\title{
Algebraic method for group classification of (1+1)-dimensional linear Schrödinger equations
}

\author{
Célestin Kurujyibwami ${ }^{\dagger}$, Peter Basarab-Horwath ${ }^{\ddagger}$ and Roman O. Popovych ${ }^{\S}$ \\ ${ }^{\dagger}$ College of Science and Technology, University of Rwanda, P.O. Box: 3900, Kigali, Rwanda \\ Linköping University, 58183 Linköping, Sweden \\ E-mail: celeku@yahoo.fr \\ ¥ Linköping University, 58183 Linköping, Sweden \\ E-mail: pehor@mail.liu.se \\ $\S$ Wolfgang Pauli Institute, Oskar-Morgenstern-Platz 1, A-1090 Vienna, Austria \\ Institute of Mathematics of NAS of Ukraine, 3 Tereshchenkivs'ka Str., 01004 Kyiv, Ukraine \\ E-mail: rop@imath.kiev.ua
}

\begin{abstract}
We carry out the complete group classification of the class of $(1+1)$-dimensional linear Schrödinger equations with complex-valued potentials. After introducing the notion of uniformly semi-normalized classes of differential equations, we compute the equivalence groupoid of the class under study and show that it is uniformly semi-normalized. More specifically, each admissible transformation in the class is the composition of a linear superposition transformation of the corresponding initial equation and an equivalence transformation of this class. This allows us to apply the new version of the algebraic method based on uniform semi-normalization and reduce the group classification of the class under study to the classification of low-dimensional appropriate subalgebras of the associated equivalence algebra. The partition into classification cases involves two integers that characterize Lie symmetry extensions and are invariant with respect to equivalence transformations.
\end{abstract}

\section{Introduction}

A standard assumption of quantum mechanics requires that the Hamiltonian of a quantum system be Hermitian since this guarantees that the energy spectrum is real and that the time evolution of the system is unitary and hence probability-preserving [10. For linear Shrödinger equations, this means that only equations with real-valued potentials are considered to be physically relevant. Since the above assumption is, unlike the other axioms of quantum mechanics, more mathematical than physical, attempts at weakening or modifying the Hermitian property of Hamiltonians have recently been made by looking at so-called $\mathcal{P} \mathcal{T}$-symmetric Hamiltonians [9, 10, 25]. Here $\mathcal{P}$ is the space reflection (or parity) operator and $\mathcal{T}$ is the time reversal operator. Some complex potentials are associated with non-Hermitian $\mathcal{P} \mathcal{T}$-symmetric Hamiltonians. Non-Hermitian $\mathcal{P} \mathcal{T}$-symmetric Hamiltonians have been used to describe (observable) phenomena in quantum mechanics, such as systems interacting with electromagnetic fields, dissipative processes such as radioactive decay, the ground state of Bose systems of hard spheres and both bosonic and fermionic degrees of freedom. Other important applications of non-Hermitian $\mathcal{P} \mathcal{T}$ symmetric Hamiltonians are to be found in scattering theory which include numerical investigations of various physical phenomena in optics, condensed matter physics, scalar wave equations (acoustical scattering) and Maxwell's equations (electromagnetic scattering), in quasi-exactly solvable Hamiltonians, complex crystals and quantum field theory [9, 10, 25]. In general, however, the physical interpretation of linear Schrödinger equations with complex potentials is not completely clear.

The study of Lie symmetries of Schrödinger equations was begun in the early 1970's after the revival of Lie's classical methods (see for instance [34]). Lie symmetries of the free $(1+3)$ dimensional Schrödinger equations were first considered in [27. Therein it was suggested to 
call the essential part of the maximal Lie symmetry group of the free Schrödinger equation the Schrödinger group. In [28] it was noted that the results of [27] could be extended directly to any number of space variables, and the isomorphism of the Lie symmetry groups of the Schrödinger equations of the $n$-dimensional harmonic oscillator and of the $n$-dimensional free fall to the symmetry group for the $(1+n)$-dimensional free Schrödinger equation was proved in [28, 29, 30]. This gave a hint for the construction of point transformations connecting these equations. The problem of finding Lie symmetries of $(1+n)$-dimensional linear Schrödinger equations with real-valued potentials was considered in [11, 30]. In particular, in [30] the general "potential-independent" form of point symmetry transformations of these equations was found under the a priori assumption of fibre preservation. The classifying equation involving both transformation components and the potential was derived and used to obtain an upper bound of dimensions of Lie symmetry groups admitted by linear Schrödinger equations. Then some static potentials of physical relevance were considered, including the harmonic oscillator, the free fall, the inverse square potential, the anisotropic harmonic oscillator and the time-dependent Kepler problem. The case of arbitrary time-independent real-valued potential was studied in [11. Although it was claimed there that "the general solution and a complete list of such potentials and their symmetry groups are then given for the cases $n=1,2,3$ ", it is now considered that this list is not complete. Note that in the papers cited above, phase translations and amplitude scalings were ignored, which makes certain points inconsistent.

Similar studies were carried out for the time-dependent Schrödinger equation for the twodimensional harmonic oscillator and for the two- and three-dimensional hydrogen-like atom in [1, 2. Closely related research on both first- and higher-order symmetry operators of linear Schrödinger equations and separation variables for such equations was initiated in the same time (see [24] and references therein).

After this "initial" stage of research into linear Schrödinger equations, the study of Lie symmetries was extended to various nonlinear Schrödinger equations [12, 14, 15, 16, 17, 18, 19, 26, 37, 38, 43. However, the group classification of linear Schrödinger equations with arbitrary complex-valued potentials still remains an open problem.

Our philosophy is that symmetries underlie physical theories and that it is therefore reasonable to look for physically relevant models from a set of models (with undetermined parameters) using symmetry criteria. The selection of possible models is made first by solving the group classification problem for the (class of) models at hand and then choosing a suitable model (or set of models) from the list of models obtained in the classification procedure. This procedure consists essentially of two parts: given a parameterized class of models, first determine the symmetry group that is common for all models from the class and then describe models admitting symmetry groups that are extensions of this common symmetry group [34].

In this paper we carry out the group classification of $(1+1)$-dimensional linear Schrödinger equations with complex-valued potentials, having the general form

$$
i \psi_{t}+\psi_{x x}+V(t, x) \psi=0
$$

where $\psi$ is an unknown complex-valued function of two real independent variables $t$ and $x$ and $V$ is an arbitrary smooth complex-valued potential also depending on $t$ and $x$. To achieve this, we apply the algebraic method of group classification (which we describe further on in this paper) and reduce the problem of the group classification of the class (10) to the classification of appropriate subalgebras of the associated equivalence algebra [6, 39]. In order to reduce the standard form of Schrödinger equations to the form (11), we scale $t$ and $x$ and change the sign of $V$. Note that the larger class of $(1+1)$-dimensional linear Schrödinger equations with "real" variable mass $m=m(t, x) \neq 0$ can be mapped to the class (11) by a family of point equivalence transformations in a way similar to that of gauging coefficients in linear evolution equations, cf. [21, 34, 40]. Hence the group classification of the class (11) also provides the group classification of this larger class. 
A particular feature of the above equations is that the independent variables $t, x$, on the one hand, and the dependent variable $\psi$ and arbitrary element $V$, on the other hand, belong to different fields. This feature needs a delicate treatment of objects involving $\psi$ or $V$. It is possible to consider Schrödinger equations from a "real perspective" by representing them as systems of two equations for the real and the imaginary parts of $\psi$, but such a representation will only complicate the whole discussion. The use of the absolute value and the argument of $\psi$ instead of the real and the imaginary parts is even less convenient since it leads to nonlinear systems instead of linear ones. This is why we work with complex-valued functions. We then need to formally extend the space of variables $(t, x, \psi)$ with $\psi^{*}$ and the space of the arbitrary element $V$ with $V^{*}$. Here and in what follows star denotes the complex conjugate. In particular, we consider $\psi^{*}$ (resp. $V^{*}$ ) as an argument for all functions depending on $\psi$ (resp. $V$ ), including components of point transformations and of vector fields. When we restrict a differential function of $\psi$ to the solution set of an equation from the class (1), we also take into account the complex conjugate of the equation, that is $-i \psi_{t}^{*}+\psi_{x x}^{*}+V^{*}(t, x) \psi^{*}=0$. However, it is sufficient to test invariance and equivalence conditions only for the original equations since the results of this testing will be the same for their complex conjugate counterparts. Presenting point transformations, we omit the transformation components for $\psi^{*}$ and $V^{*}$ since they are obtained by conjugating those for $\psi$ and $V$.

The structure of this paper is the following: In Section 2 we describe the general framework of the group classification of classes of differential equations. We define various objects related to point transformations and discuss their properties. In Section 3 we extend the algebraic method of group classification to uniformly semi-normalized classes of differential equations. We compute the equivalence groupoid, the equivalence group and the equivalence algebra of the class (11) in Section 4. It turns out that the class (1) has rather good transformational properties: it is uniformly semi-normalized with respect to linear superposition of solutions. In Section 5 we then analyze the determining equations for the Lie symmetries of equations from the class (11), find the kernel Lie invariance algebra of this class and single out the classifying condition for admissible Lie symmetry extensions. In Section 6 we study properties of appropriate subalgebras of the equivalence algebra, classify them and complete the group classification of the class (11). In Section 7 we illustrate the advantages of the algebraic method of group classification by performing the group classification of the class (1) in a different way. The group classification of $(1+1)$-dimensional linear Schrödinger equations with real potentials is presented in Section 8 . In the final section we summarize results of the paper.

\section{Group classification in classes of differential equations}

In this section we give the definitions and notation needed for the group classification of differential equations. For more details see [6, 7, 32, 34, 39.

We begin with a definition of the notion of class of differential equations. Let $\mathcal{L}_{\theta}$ be a system $L\left(x, u_{(p)}, \theta_{(q)}\left(x, u_{(p)}\right)\right)=0$ of $l$ differential equations $L^{1}=0, \ldots, L^{l}=0$ parameterized by a tuple of arbitrary elements $\theta\left(x, u_{(p)}\right)=\left(\theta^{1}\left(x, u_{(p)}\right), \ldots, \theta^{k}\left(x, u_{(p)}\right)\right)$, where $x=\left(x_{1}, \ldots, x_{n}\right)$ is the tuple of independent variables and $u_{(p)}$ is the set of the dependent variables $u=\left(u^{1}, \ldots, u^{m}\right)$ together with all derivatives of $u$ with respect to $x$ of order less than or equal to $p$. The symbol $\theta_{(q)}$ stands for the set of partial derivatives of $\theta$ of order less than or equal to $q$ with respect to the variables $x$ and $u_{(p)}$. The tuple of arbitrary elements $\theta$ runs through the set $\mathcal{S}$ of solutions of an auxiliary system of differential equations $S\left(x, u_{(p)}, \theta_{\left(q^{\prime}\right)}\left(x, u_{(p)}\right)\right)=0$ and differential inequalities $\Sigma\left(x, u_{(p)}, \theta_{\left(q^{\prime}\right)}\left(x, u_{(p)}\right)\right) \neq 0$ (other kinds of inequalities may also appear here), in which both $x$ and $u_{(p)}$ play the role of independent variables and $S$ and $\Sigma$ are tuples of smooth functions depending on $x, u_{(p)}$ and $\theta_{\left(q^{\prime}\right)}$. The set $\left\{\mathcal{L}_{\theta} \mid \theta \in \mathcal{S}\right\}=:\left.\mathcal{L}\right|_{\mathcal{S}}$ is called a class (of systems) of differential equations that is defined by the parameterized form of systems $\mathcal{L}_{\theta}$ and the set $\mathcal{S}$ run by the arbitrary elements $\theta$. 
Thus, for the class (10) we have two partial differential equations (including the complex conjugate equation) for two (formally unrelated) dependent variables $\psi$ and $\psi^{*}$ of two independent variables $t$ and $x$, and two (formally unrelated) arbitrary elements $\theta=\left(V, V^{*}\right)$, which depend only on $t$ and $x$. Therefore, the auxiliary system for the arbitrary elements of the class (10) is

$$
\begin{aligned}
& V_{\psi}=V_{\psi^{*}}=V_{\psi_{t}}=V_{\psi_{t}^{*}}=V_{\psi_{x}}=V_{\psi_{x}^{*}}=V_{\psi_{t t}}=V_{\psi_{t t}^{*}}=V_{\psi_{t x}}=V_{\psi_{t x}^{*}}=V_{\psi_{x x}}=V_{\psi_{x x}^{*}}=0, \\
& V_{\psi}^{*}=V_{\psi^{*}}^{*}=V_{\psi_{t}}^{*}=V_{\psi_{t}^{*}}^{*}=V_{\psi_{x}}^{*}=V_{\psi_{x}^{*}}^{*}=V_{\psi_{t t}}^{*}=V_{\psi_{t t}^{*}}^{*}=V_{\psi_{t x}}^{*}=V_{\psi_{t x}^{*}}^{*}=V_{\psi_{x x}}^{*}=V_{\psi_{x x}^{*}}^{*}=0 .
\end{aligned}
$$

For a class of differential equations $\left.\mathcal{L}\right|_{\mathcal{S}}$, there are objects of various structures that consist of point transformations related to this class. Let $\mathcal{L}_{\theta}$ and $\mathcal{L}_{\tilde{\theta}}$ be systems belonging to $\left.\mathcal{L}\right|_{\mathcal{S}}$. We denote by $\mathrm{T}(\theta, \tilde{\theta})$ the set of point transformations in the space of the variables $(x, u)$ that map $\mathcal{L}_{\theta}$ to $\mathcal{L}_{\tilde{\theta}}$.

An admissible transformation of the class $\left.\mathcal{L}\right|_{\mathcal{S}}$ is a triple $(\theta, \tilde{\theta}, \varphi)$ consisting of two arbitrary elements $\theta, \tilde{\theta} \in \mathcal{S}$ such that $\mathrm{T}(\theta, \tilde{\theta}) \neq \varnothing$ and a point transformation $\varphi \in \mathrm{T}(\theta, \tilde{\theta})$. The set of all admissible transformations of the class $\left.\mathcal{L}\right|_{\mathcal{S}}$,

$$
\mathcal{G}^{\sim}=\mathcal{G}^{\sim}\left(\left.\mathcal{L}\right|_{\mathcal{S}}\right):=\{(\theta, \tilde{\theta}, \varphi) \mid \theta, \tilde{\theta} \in \mathcal{S}, \varphi \in \mathrm{T}(\theta, \tilde{\theta})\}
$$

has the structure of a groupoid: for any $\theta \in \mathcal{S}$ the triple $(\theta, \theta$,id), where id is the identity point transformation, is an element of $\mathcal{G}^{\sim}$, every $(\theta, \tilde{\theta}, \varphi) \in \mathcal{G}^{\sim}$ is invertible and $\mathcal{G}^{\sim}$ is closed under composition. This is why the set $\mathcal{G}^{\sim}$ is called the equivalence groupoid of the class $\left.\mathcal{L}\right|_{\mathcal{S}}$.

The (usual) equivalence (pseudo)group $G^{\sim}=G^{\sim}\left(\left.\mathcal{L}\right|_{\mathcal{S}}\right.$ ) of the class $\left.\mathcal{L}\right|_{\mathcal{S}}$ is defined as being the set of point transformations in the joint space of independent and dependent variables, their derivatives and arbitrary elements with local coordinates $\left(x, u_{(p)}, \theta\right)$ that are projectable to the space of $\left(x, u_{\left(p^{\prime}\right)}\right)$ for any $0 \leqslant p^{\prime} \leqslant p$, preserve the contact structure on the space with local coordinates $\left(x, u_{(p)}\right)$, and map every system from the class $\left.\mathcal{L}\right|_{\mathcal{S}}$ to a system from the same class. Elements of the group $G^{\sim}$ are called equivalence transformations. This definition includes two fundamental conditions for general equivalence transformations: the preservation of the class $\left.\mathcal{L}\right|_{\mathcal{S}}$ and the preservation of the contact structure on the space with local coordinates $\left(x, u_{(p)}\right)$. The conditions of projectability and locality with respect to arbitrary elements can be weakened, and this leads to various generalizations of the notion of equivalence group (see [39]). Note that each equivalence transformation $\mathcal{T} \in G^{\sim}$ generates a family of admissible transformations from $\mathcal{G}^{\sim}$, $G^{\sim} \ni \mathcal{T} \rightarrow\left\{\left(\theta, \mathcal{T} \theta,\left.\mathcal{T}\right|_{(x, u)}\right) \mid \theta \in \mathcal{S}\right\} \subset \mathcal{G}^{\sim}$, where $\left.\mathcal{T}\right|_{(x, u)}$ is the restriction of $\mathcal{T}$ to the space of $(x, u)$. For a generalized equivalence group, the restriction of $\mathcal{T}$ is made after fixing a value of $\theta$, which can be denoted as $\left.\mathcal{T}^{\theta}\right|_{(x, u)}$.

The equivalence group of a subclass $\left.\mathcal{L}\right|_{\mathcal{S}^{\prime}}, \mathcal{S}^{\prime} \subset \mathcal{S}$, of the class $\left.\mathcal{L}\right|_{\mathcal{S}}$ is called a conditional equivalence group of the class $\left.\mathcal{L}\right|_{\mathcal{S}}$ that is associated with the subclass $\left.\mathcal{L}\right|_{\mathcal{S}^{\prime}}$. A useful way of describing the equivalence groupoid $\mathcal{G}^{\sim}$ is to classify maximal conditional equivalence groups of the class $\left.\mathcal{L}\right|_{\mathcal{S}}$ up to $G^{\sim}$-equivalence and then to classify (up to an appropriate conditional equivalence) the admissible transformations that are not generated by conditional equivalence transformations (see [39] for more details).

The equivalence algebra $\mathfrak{g}^{\sim}=\mathfrak{g}^{\sim}\left(\left.\mathcal{L}\right|_{\mathcal{S}}\right)$ of the class $\left.\mathcal{L}\right|_{\mathcal{S}}$ is defined as the set of generators of one-parameter groups of equivalence transformations of the class $\left.\mathcal{L}\right|_{\mathcal{S}}$. These generators are vector fields in the space of $\left(x, u_{(p)}, \theta\right)$, that are projectable to the space of $\left(x, u_{\left(p^{\prime}\right)}\right)$ for any $0 \leqslant p^{\prime} \leqslant p$ and whose projections to the space of $\left(x, u_{(p)}\right)$ are the $p$ th order prolongations of the corresponding projections to the space of $(x, u)$.

The maximal point symmetry (pseudo)group $G_{\theta}$ of the system $\mathcal{L}_{\theta}$ (for a fixed $\theta \in \mathcal{S}$ ) is a (pseudo)group of transformations that act in the space of independent and dependent variables that preserve the solution set of the system $\mathcal{L}_{\theta}$. Each $G_{\theta}$ can be interpreted as a vertex group of the equivalence groupoid $\mathcal{G}^{\sim}$. The intersection $G^{\cap}=G^{\cap}\left(\left.\mathcal{L}\right|_{\mathcal{S}}\right):=\bigcap_{\theta \in \mathcal{S}} G_{\theta}$ of all $G_{\theta}, \theta \in \mathcal{S}$, is called the kernel of the maximal point symmetry groups of systems from $\left.\mathcal{L}\right|_{\mathcal{S}}$. 
The vector fields in the space of $(x, u)$ generating one-parameter subgroups of the maximal point symmetry group $G_{\theta}$ of the system $\mathcal{L}_{\theta}$ form a Lie algebra $\mathfrak{g}_{\theta}$ with the Lie bracket defined by commutators of vector fields. It is called the maximal Lie invariance algebra of $\mathcal{L}_{\theta}$. The kernel invariance algebra of the class $\left.\mathcal{L}\right|_{\mathcal{S}}$ is the intersection $\mathfrak{g}^{\cap}=\mathfrak{g}^{\cap}\left(\left.\mathcal{L}\right|_{\mathcal{S}}\right):=\bigcap_{\theta \in \mathcal{S}} \mathfrak{g}_{\theta}$ of the algebras $\mathfrak{g}_{\theta}, \theta \in \mathcal{S}$.

The classical group classification problem for the class $\left.\mathcal{L}\right|_{\mathcal{S}}$ is to list all $G^{\sim}$-inequivalent values of $\theta \in \mathcal{S}$ for which the corresponding maximal Lie invariance algebras, $\mathfrak{g}_{\theta}$, are larger than the kernel invariance algebra $\mathfrak{g}^{\cap}$. There may be additional point equivalences between the cases obtained in this way and these additional equivalences have then to be incorporated into the results. This solves the group classification problem for the class $\left.\mathcal{L}\right|_{\mathcal{S}}$ up to $\mathcal{G}^{\sim}$-equivalence.

Summing up, objects to be found in the course of group classification of the class $\left.\mathcal{L}\right|_{\mathcal{S}}$ include the equivalence groupoid $\mathcal{G}^{\sim}$, the equivalence group $G^{\sim}$, the equivalence algebra $\mathfrak{g}^{\sim}$, the kernel invariance algebra $\mathfrak{g}^{\cap}$ and a complete list of $G^{\sim}$-inequivalent (resp. $\mathcal{G}^{\sim}$-inequivalent) values of $\theta$ with the corresponding Lie symmetry extensions of $\mathfrak{g}^{\cap}$. Additional point equivalences between classification cases can be computed directly via looking for pairs of cases with similar Lie invariance algebras (if two systems are equivalent under an invertible point transformation, then their Lie symmetry algebras are isomorphic). Therefore, the construction of the equivalence groupoid $\mathcal{G}^{\sim}$ can be excluded from the procedure of group classification if this groupoid is of complicated structure, e.g., due to the involved hierarchy of maximal conditional equivalence groups of the class $\left.\mathcal{L}\right|_{\mathcal{S}}$.

The classical way of performing a group classification of a class $\left.\mathcal{L}\right|_{\mathcal{S}}$ is to use the infinitesimal invariance criterion [31, 34]: under an appropriate nondegeneracy condition for the system $\left.\mathcal{L}_{\theta} \in \mathcal{L}\right|_{\mathcal{S}}$, a vector field $Q=\xi^{j}(x, u) \partial_{x_{j}}+\eta^{a}(x, u) \partial_{u^{a}}$ belongs to the maximal Lie invariance algebra $\mathfrak{g}_{\theta}$ of $\mathcal{L}_{\theta}$ if and only if the condition

$$
Q_{(p)} L\left(x, u_{(p)}, \theta_{(q)}\left(x, u_{(p)}\right)\right)=0,
$$

holds on the manifold $\mathcal{L}_{\theta}^{p}$ defined by the system $\mathcal{L}_{\theta}$ together with its differential consequences in the jet space $J^{(p)}$. Here the indices $j$ and $a$ run from 1 to $n$ and from 1 to $m$, respectively, and we use the summation convention for repeated indices. $Q_{(p)}$ denotes the standard $p$ th prolongation of the vector field $Q$,

$$
Q_{(p)}=Q+\sum_{0<|\alpha| \leqslant p}\left(D_{1}^{\alpha_{1}} \cdots D_{n}^{\alpha_{n}}\left(\eta^{a}-\xi^{j} u_{j}^{a}\right)+\xi^{j} u_{\alpha+\delta_{j}}^{a}\right) \partial_{u_{\alpha}^{a}} .
$$

The tuple $\alpha=\left(\alpha_{1}, \ldots, \alpha_{n}\right)$ is a multiindex, $\alpha_{j} \in \mathbb{N} \cup 0,|\alpha|:=\alpha_{1}+\cdots+\alpha_{n}$, and $\delta_{j}$ is the multiindex whose $i$ th entry equals 1 and whose other entries are zero. The variable $u_{\alpha}^{a}$ of the jet space $J^{(p)}$ is identified with the derivative $\partial^{|\alpha|} u^{a} / \partial x_{1}^{\alpha_{1}} \ldots \partial x_{n}^{\alpha_{n}} . D_{j}=\partial_{j}+u_{\alpha+\delta_{j}}^{a} \partial_{u_{\alpha}^{a}}$ is the total derivative operator with respect to the variable $x_{j}$.

The infinitesimal invariance criterion yields the system of determining equations for the components of the generators of the one-parameter Lie symmetry groups of systems from the class $\left.\mathcal{L}\right|_{\mathcal{S}}$, where the arbitrary elements $\theta$ play the role of parameters. Integrating those determining equations that do not involve arbitrary elements gives a preliminary form of the generator components, and one must then solve the remaining equations. The solution of these remaining equations depends on the values of the arbitrary elements. We call these equations the classifying equations for the class $\left.\mathcal{L}\right|_{\mathcal{S}}$.

In order to find the kernel invariance algebra $\mathfrak{g}^{\cap}$, one must first split the determining equations with respect to parametric derivatives of arbitrary elements and of dependent variables and then solve the system obtained.

Finding Lie symmetry extensions of the kernel Lie algebra depends on an analysis of the classifying equations. This part of solving the group classification problem is intricate and various techniques are used to obtain the solution. There are two main approaches. If the class 
considered has a simple structure (for example, when arbitrary elements are constants or are functions of just one argument), then the techniques used rely on the study of the compatibility of the classifying equations and their direct solution with respect to both the components of Lie symmetry generators and the arbitrary elements (up to the equivalence defined by the equivalence group). See, for instance, [8, 34, 36, 41, 42] and the references given there. For more complicated classes, the direct approach seems to be irrelevant, and more advanced algebraic techniques need to be used.

\section{Uniformly semi-normalized classes}

In the most general setting, the main point of the algebraic approach to group classification is to classify (up to certain equivalence relation induced by point transformations between systems belonging to the class $\left.\mathcal{L}\right|_{\mathcal{S}}$ under study) certain Lie algebras of vector fields related to these systems 1 The key problem is to select sets of vector fields to be classified and the equivalence relation to be used in this classification [6]. For the application of the algebraic method to be effective, the selected objects have to satisfy certain consistency conditions which then require particular properties of the equivalence groupoid $\mathcal{G}^{\sim}$ of $\left.\mathcal{L}\right|_{\mathcal{S}}$. To this end, we begin with some definitions which enable us to formulate our approach.

We say that the class $\left.\mathcal{L}\right|_{\mathcal{S}}$ is normalized if its equivalence groupoid $\mathcal{G}^{\sim}$ is generated by its equivalence group $G^{\sim}$. We say that it is semi-normalized if the equivalence groupoid $\mathcal{G}^{\sim}$ is generated by transformations from $G^{\sim}$ and point symmetry transformations of the corresponding source or target systems. It is clear that any normalized class of differential equations is seminormalized. Normalized classes are especially convenient when one applies the algebraic method of group classification. If the class $\left.\mathcal{L}\right|_{\mathcal{S}}$ is normalized, then the Lie symmetry extensions of its kernel invariance algebra are obtained via the classification of appropriate subalgebras of the equivalence algebra whose projections onto the space with local coordinates $(x, u)$ coincide with the maximal Lie invariance algebras of systems from $\left.\mathcal{L}\right|_{\mathcal{S}}$. The property of semi-normalization is useful for determining equivalences between Lie symmetry extensions but not for finding such extensions. For rigorous definitions and more details, we refer the reader to [6, 39].

Classes of differential equations that are not normalized but have stronger normalization properties than semi-normalization often appear in physical applications. This is why it is important to weaken the normalization property in such a way that still allows us to apply group classification techniques analogous to those developed for normalized classes.

Definition 1. Given a class of differential equations $\left.\mathcal{L}\right|_{\mathcal{S}}$ with equivalence groupoid $\mathcal{G}^{\sim}$ and (usual) equivalence group $G^{\sim 2}$ suppose that for each $\theta \in \mathcal{S}$ the point symmetry group $G_{\theta}$ of the system $\left.\mathcal{L}_{\theta} \in \mathcal{L}\right|_{\mathcal{S}}$ contains a subgroup $N_{\theta}$ such that the family $\mathcal{N}_{\mathcal{S}}=\left\{N_{\theta} \mid \theta \in \mathcal{S}\right\}$ of all these subgroups satisfies the following properties:

1. $\left.\mathcal{T}\right|_{(x, u)} \notin N_{\theta}$ for any $\theta \in \mathcal{S}$ and any $\mathcal{T} \in G^{\sim}$ with $\mathcal{T} \neq$ id.

2. $N_{\mathcal{T} \theta}=\left.\mathcal{T}\right|_{(x, u)} N_{\theta}\left(\left.\mathcal{T}\right|_{(x, u)}\right)^{-1}$ for any $\theta \in \mathcal{S}$ and any $\mathcal{T} \in G^{\sim}$.

3. For any $\left(\theta^{1}, \theta^{2}, \varphi\right) \in \mathcal{G}^{\sim}$ there exist $\varphi^{1} \in N_{\theta^{1}}, \varphi^{2} \in N_{\theta^{2}}$ and $\mathcal{T} \in G^{\sim}$ such that $\theta^{2}=\mathcal{T} \theta^{1}$ and $\varphi=\varphi^{2}\left(\left.\mathcal{T}\right|_{(x, u)}\right) \varphi^{1}$.

Here $\left.\mathcal{T}\right|_{(x, u)}$ denotes the restriction of $\mathcal{T}$ to the space with local coordinates $(x, u)$. We then say that the class of differential equations $\left.\mathcal{L}\right|_{\mathcal{S}}$ is uniformly semi-normalized with respect to the symmetry-subgroup family $\mathcal{N}_{\mathcal{S}}$.

\footnotetext{
${ }^{1}$ See, e.g., [3, 4, 19, 20, 23, 37, 38, 44, and 6, 7, 32, 39] for the application of the preliminary and advanced versions of the algebraic method, respectively, to various classes of differential equations.

${ }^{2} \mathrm{~A}$ subgroup of the equivalence group can be considered here instead of the entire group.
} 
The qualification "uniformly" is justified by the fact that in practically relevant examples of such classes all the subgroups $N_{\theta}$ 's are isomorphic or at least of a similar structure (in particular, of the same dimension). The first property in Definition 1 means that the intersection of each subgroup $N_{\theta}$ with the restriction of $G^{\sim}$ to the space of $(x, u)$ is just the identity transformation. The second property can be interpreted as equivariance of equivalence transformations with respect to $\mathcal{N}_{\mathcal{S}}$. The third property means, essentially, that the entire equivalence groupoid $\mathcal{G}^{\sim}$ is generated by equivalence transformations and transformations from uniform point symmetry groups. One of the symmetry transformations $\varphi^{1}$ or $\varphi^{2}$ in the last property may be taken to be the identity.

Each normalized class of differential equations is uniformly semi-normalized with respect to the trivial family $\mathcal{N}_{\mathcal{S}}$, where for each $\theta$ the group $N_{\theta}$ consists of just the identity transformation. It is also obvious that each uniformly semi-normalized class is semi-normalized. At the same time, there exist semi-normalized classes that are not uniformly semi-normalized. A simple example of such a class is given by the class ND of nonlinear diffusion equations of the form $u_{t}=\left(f(u) u_{x}\right)_{x}$ with $f_{u} \neq 0$, which is a classic example in the group analysis of differential equations [33, 34]. Such equations with special power nonlinearities of the form $f=c_{1}\left(u+c_{0}\right)^{-4 / 3}$ have singular symmetry properties within the class ND. This fact does not allow the class ND to be normalized, although it is semi-normalized. The elements from $\mathcal{G}^{\sim}(\mathrm{ND})$ that are not generated by elements of $G^{\sim}(\mathrm{ND})$ are given by the equivalence transformations of equations with the above power nonlinearities that are composed with conformal symmetry transformations of these equations. The nonlinear diffusion equation with $f=c_{1}\left(u+c_{0}\right)^{-4 / 3}$ admits the conformal symmetry group with infinitesimal generator $x^{2} \partial_{x}-3 x\left(u+c_{0}\right) \partial_{u}$, but this is not a normal subgroup of the point symmetry group of the equation.

The following result, which we call the theorem on splitting symmetry groups in uniformly semi-normalized classes, provides a theoretical basis for the algebraic method of group classification of such classes.

Theorem 2. Let a class of differential equations $\left.\mathcal{L}\right|_{\mathcal{S}}$ be uniformly semi-normalized with respect to a symmetry-subgroup family $\mathcal{N}_{\mathcal{S}}=\left\{N_{\theta} \mid \theta \in \mathcal{S}\right\}$. Then for each $\theta \in \mathcal{S}$ the point symmetry group $G_{\theta}$ of the system $\left.\mathcal{L}_{\theta} \in \mathcal{L}\right|_{\mathcal{S}}$ splits over $N_{\theta}$. More specifically, $N_{\theta}$ is a normal subgroup of $G_{\theta}, G_{\theta}^{\text {ess }}=\left.G^{\sim}\right|_{(x, u)} \cap G_{\theta}$ is a subgroup of $G_{\theta}$, and the group $G_{\theta}$ is the semidirect product of $G_{\theta}^{\text {ess }}$ acting on $N_{\theta}, G_{\theta}=G_{\theta}^{\text {ess }} \ltimes N_{\theta}$. Here $\left.G^{\sim}\right|_{(x, u)}$ denotes the restriction of $G^{\sim}$ to the space with local coordinates $(x, u),\left.G^{\sim}\right|_{(x, u)}=\left\{\left.\mathcal{T}\right|_{(x, u)} \mid \mathcal{T} \in G^{\sim}\right\}$.

Proof. We fix an arbitrary $\theta \in \mathcal{S}$ and take an arbitrary $\varphi \in G_{\theta}$. Then $(\theta, \theta, \varphi) \in \mathcal{G}^{\sim}$ and, by the third property in Definition 1, the transformation $\varphi$ admits the factorization $\varphi=\left.\mathcal{T}\right|_{(x, u)} \varphi^{1}$ for some $\mathcal{T} \in G^{\sim}$ and some $\varphi^{1} \in N_{\theta}$. The element $N_{\theta}$ of the family $\mathcal{N}_{\mathcal{S}}$ is a subgroup of $G_{\theta}, N_{\theta}<G_{\theta}$ and hence the transformation $\varphi^{0}:=\left.\mathcal{T}\right|_{(x, u)}=\varphi\left(\varphi^{1}\right)^{-1}$ also belongs to $G_{\theta}$, and consequently to $\left.G^{\sim}\right|_{(x, u)} \cap G_{\theta}=: G_{\theta}^{\text {ess }}$, which is a subgroup of $G_{\theta}$ as it is the intersection of two groups. From this, it follows that for any $\varphi \in G_{\theta}$ we have the representation $\varphi=\varphi^{0} \varphi^{1}$, where $\varphi^{0} \in G_{\theta}^{\text {ess }}$ and $\varphi^{1} \in N_{\theta}$.

The first property of Definition 1 means that the intersection of $\left.G^{\sim}\right|_{(x, u)}$ and $N_{\theta}$ consists of just the identity transformation so that the intersection $G_{\theta}^{\text {ess }} \cap N_{\theta}$ contains only the identity transformation.

For an arbitrary $\varphi \in G_{\theta}$ and an arbitrary $\tilde{\varphi} \in N_{\theta}$, we consider the composition $\varphi \tilde{\varphi} \varphi^{-1}$. As an element of $G_{\theta}$, the transformation $\varphi$ admits the factorization $\varphi=\varphi^{0} \varphi^{1}$ with some $\varphi^{0} \in G_{\theta}^{\text {ess }}$ and some $\varphi^{1} \in N_{\theta}$. Since $G_{\theta}^{\text {ess }}<\left.G^{\sim}\right|_{(x, u)}$, there exists a $\mathcal{T} \in G^{\sim}$ such that $\mathcal{T} \theta=\theta$ and $\varphi^{0}=\left.\mathcal{T}\right|_{(x, u)}$. By property 2 of Definition [1, we obtain $N_{\theta}=\varphi^{0} N_{\theta}\left(\varphi^{0}\right)^{-1}$. Hence the composition $\varphi \tilde{\varphi} \varphi^{-1}=\varphi^{0} \varphi^{1} \tilde{\varphi}\left(\varphi^{1}\right)^{-1}\left(\varphi^{0}\right)^{-1}$ belongs to $N_{\theta}$. Thus we have that $N_{\theta}$ is a normal subgroup of $G_{\theta}$, $N_{\theta} \triangleleft G_{\theta}$, and so $G_{\theta}=G_{\theta}^{\text {ess }} \ltimes N_{\theta}$.

The members of the family $\mathcal{N}_{\mathcal{S}}=\left\{N_{\theta} \mid \theta \in \mathcal{S}\right\}$ are called uniform point symmetry groups of the systems from the class $\left.\mathcal{L}\right|_{\mathcal{S}}$, and the subgroup $G_{\theta}^{\text {ess }}$ is called the essential point symmetry 
group of the system $\mathcal{L}_{\theta}$ associated with the uniform point symmetry group $N_{\theta}$. The knowledge of a family of uniform point symmetry groups trivializes them in the following sense: since $G_{\theta}$ splits over $N_{\theta}$ for each $\theta$, then we only need to find the subgroup $G_{\theta}^{\text {ess }}$ in order to construct $G_{\theta}$.

The infinitesimal version of Theorem 2 may be called the theorem on splitting invariance algebras in uniformly semi-normalized classes. This version follows immediately from Theorem 2 if we replace the groups with the corresponding algebras of generators of the one-parameter subgroups of these groups.

Theorem 3. Suppose that a class of differential equations $\left.\mathcal{L}\right|_{\mathcal{S}}$ is uniformly semi-normalized with respect to a family of symmetry subgroups $\mathcal{N}_{\mathcal{S}}=\left\{N_{\theta} \mid \theta \in \mathcal{S}\right\}$. Then for each $\theta \in \mathcal{S}$ the Lie algebras $\mathfrak{g}_{\theta}^{\text {ess }}$ and $\mathfrak{n}_{\theta}$ that are associated with the groups $G_{\theta}^{\text {ess }}$ and $N_{\theta}$ are, respectively, a subalgebra and an ideal of the maximal Lie invariance algebra $\mathfrak{g}_{\theta}$ of the system $\left.\mathcal{L}_{\theta} \in \mathcal{L}\right|_{\mathcal{S}}$. Moreover, the algebra $\mathfrak{g}_{\theta}$ is the semi-direct sum $\mathfrak{g}_{\theta}=\mathfrak{g}_{\theta}^{\text {ess }} \notin \mathfrak{n}_{\theta}$, and $\mathfrak{g}_{\theta}^{\text {ess }}=\left.\mathfrak{g}^{\sim}\right|_{(x, u)} \cap \mathfrak{g}_{\theta}$, where $\left.\mathfrak{g}^{\sim}\right|_{(x, u)}$ denotes the restriction of $\mathfrak{g}^{\sim}$ to the space with local coordinates $(x, u)$.

The group classification problem for a uniformly semi-normalized class $\left.\mathcal{L}\right|_{\mathcal{S}}$ is solved in the following way: when computing the equivalence groupoid $\mathcal{G}^{\sim}$ and analyzing its structure, we construct a family of uniform point symmetry groups $\mathcal{N}_{\mathcal{S}}=\left\{N_{\theta} \mid \theta \in \mathcal{S}\right\}$, which then establishes the uniformly semi-normalization of the class $\left.\mathcal{L}\right|_{\mathcal{S}}$ and yields the corresponding uniform Lie invariance algebras $\mathfrak{n}_{\theta}$ 's. The subgroup $G_{\theta}^{\text {ess }}=\left.G^{\sim}\right|_{(x, u)} \cap G_{\theta}$ and the subalgebra $\mathfrak{g}_{\theta}^{\text {ess }}=\left.\mathfrak{g}^{\sim}\right|_{(x, u)} \cap \mathfrak{g}_{\theta}$ which are the complements of $N_{\theta}$ and $\mathfrak{n}_{\theta}$ respectively, are in general not known on this step. By Theorem 3, we have for each $\theta \in \mathcal{S}$ that the maximal Lie invariance algebra $\mathfrak{g}_{\theta}$ of the system $\mathcal{L}_{\theta}$ is given by the semi-direct sum $\mathfrak{g}_{\theta}=\mathfrak{g}_{\theta}^{\text {ess }} \in \mathfrak{n}_{\theta}$. Essential Lie invariance algebras are subalgebras of $\left.\mathfrak{g}^{\sim}\right|_{(x, u)}$ and are mapped onto each other by the differentials of restrictions of equivalence transformations: $\mathfrak{g}_{\mathcal{T} \theta}^{\text {ess }}=\left(\left.\mathcal{T}\right|_{(x, u)}\right)_{*} \mathfrak{g}_{\theta}^{\text {ess }}$. Consequently, the group classification of the class $\left.\mathcal{L}\right|_{\mathcal{S}}$ reduces to the classification of appropriate subalgebras of $\left.\mathfrak{g}^{\sim}\right|_{(x, u)}$ or, equivalently, of the equivalence algebra $\mathfrak{g}^{\sim}$ itself.

An important case of uniformly semi-normalized classes, which is relevant to the present paper, is given by classes of homogeneous linear systems of differential equations.

Consider a normalized class $\left.\mathcal{L}^{\text {inh }}\right|_{\mathcal{S} \text { inh }}$ of (generally) inhomogeneous linear systems of differential equations $\mathcal{L}_{\theta, \zeta}^{\text {inh }}$ 's of the form $L\left(x, u_{(p)}, \theta_{(q)}(x)\right)=\zeta(x)$, where $\theta$ is a tuple of arbitrary elements parameterizing the homogeneous linear left hand side and depending only on $x$ and the right hand side $\zeta$ is a tuple of arbitrary functions of $x$. Suppose that the class $\left.\mathcal{L}^{\text {inh }}\right|_{\mathcal{S}^{\text {inh }}}$ also satisfies the following conditions:

- Each system from $\left.\mathcal{L}^{\text {inh }}\right|_{\mathcal{S}^{\text {inh }}}$ is locally solvable.

- The zero function is the only common solution of the homogeneous systems from $\left.\mathcal{L}^{\text {inh }}\right|_{\mathcal{S}^{\text {inh }}}$.

- Restrictions of elements of the equivalence group $G_{\text {inh }}^{\sim}=G^{\sim}\left(\left.\mathcal{L}^{\text {inh }}\right|_{\mathcal{S}^{\text {inh }}}\right)$ to the space of $(x, u)$ are fibre-preserving transformations whose components for $u$ are affine in $u$, that is they are of the form $\tilde{x}_{j}=X^{j}(x), \tilde{u}^{a}=M^{a b}(x)\left(u^{b}+h^{b}(x)\right)$, where $\operatorname{det}\left(X_{x_{j^{\prime}}}^{j}\right) \neq 0$ and $\operatorname{det}\left(M^{a b}\right) \neq 0$.

Here the indices $j$ and $j^{\prime}$ run from 1 to $n$ and the indices $a$ and $b$ run from 1 to $m$. The functions $X^{j}$ 's and $M^{a b}$ 's may satisfy additional constraints but the $h^{a}$ 's are arbitrary smooth functions of $x$.

Any system $\mathcal{L}_{\theta \zeta}$ from the class $\left.\mathcal{L}^{\text {inh }}\right|_{\mathcal{S}^{\text {inh }}}$ is mapped to the associated homogeneous system $\mathcal{L}_{\theta 0}$ by the equivalence transformation

$$
\mathcal{T}_{\theta \zeta}: \quad \tilde{x}_{j}=x_{j}, \quad \tilde{u}^{a}=u^{a}+h^{a}(x), \quad \tilde{\theta}=\theta, \quad \tilde{\zeta}=\zeta-L\left(x, h_{(p)}(x), \theta_{(q)}(x)\right),
$$

where $h=\left(h^{1}, \ldots, h^{m}\right)$ is a solution of $\mathcal{L}_{\theta \zeta}$. In other words, the class $\left.\mathcal{L}^{\text {inh }}\right|_{\mathcal{S}^{\text {inh }}}$ is mapped, by the family $\left\{\mathcal{T}_{\theta \zeta}\right\}$ of equivalence transformations that are nonlocally parameterized by the arbitrary elements $\theta$ 's and $\zeta$ 's to the corresponding class $\left.\mathcal{L}^{\mathrm{hmg}}\right|_{\mathcal{S}^{\text {hmg }}}$ of homogeneous systems. The transformations from the equivalence group $G_{\text {inh }}^{\sim}$ with $(x, u)$-components $\tilde{x}_{j}=x_{j}, \tilde{u}^{a}=$ 
$u^{a}+h^{a}(x)$, where the $h^{a}$ 's run through the set of smooth functions of $x$, constitute a normal subgroup $N_{\text {inh }}^{\sim}$ of this group. Furthermore, $G_{\text {inh }}^{\sim}$ splits over $N_{\text {inh }}^{\sim}$ since $G_{\text {inh }}^{\sim}=H_{\text {inh }}^{\sim} \ltimes N_{\text {inh }}^{\sim}$, where $H_{\mathrm{inh}}^{\sim}$ is the subgroup of $G_{\mathrm{inh}}^{\sim}$ consisting of those elements with $h^{a}=0$. The restriction of $H_{\mathrm{inh}}^{\sim}$ to the space with local coordinates $(x, u, \theta)$ coincides with the equivalence group $G_{\mathrm{hmg}}^{\sim}$ of the class

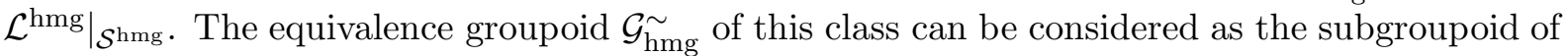
the equivalence groupoid $\mathcal{G}_{\text {inh }}^{\sim}$ of the class $\left.\mathcal{L}^{\text {inh }}\right|_{\mathcal{S}^{\text {inh }}}$ that is singled out by the constraints $\zeta=0$, $\tilde{\zeta}=0$ for the source and target systems of admissible transformations, respectively. For each relevant transformational part, the tuple of parameter-functions $h$ is an arbitrary solution of the corresponding source system. The systems $\mathcal{L}_{\theta \zeta}$ and $\mathcal{L}_{\tilde{\theta} \tilde{\zeta}}$ are $G_{\text {inh }}^{\sim}$-equivalent if and only if their homogeneous counterparts $\mathcal{L}_{\theta 0}$ and $\mathcal{L}_{\tilde{\theta} 0}$ are $G_{\mathrm{hmg}}^{\sim}$-equivalent. Thus the group classification of systems from the class $\left.\mathcal{L}^{\text {inh }}\right|_{\mathcal{S}^{\text {inh }}}$ reduces to the group classification of systems from the class $\left.\mathcal{L}^{\text {hmg }}\right|_{\mathcal{S}^{\text {hmg }}}$.

For each $\theta \in \mathcal{S}^{\text {hmg }}$ we denote by $G_{\theta 0}^{\text {lin }}$ the subgroup of the point symmetry group $G_{\theta 0}$ of $\mathcal{L}_{\theta 0}$ that consists of the linear superposition transformations:

$$
\tilde{x}_{j}=x_{j}, \quad \tilde{u}^{a}=u^{a}+h^{a}(x),
$$

where the tuple $h$ is a solution of $\mathcal{L}_{\theta 0}$. The family $\mathcal{N}_{\text {lin }}=\left\{G_{\theta 0}^{\text {lin }} \mid \theta \in \mathcal{S}_{\text {hmg }}\right\}$ of all these subgroups satisfies the properties of Definition 1. Therefore, the class $\left.\mathcal{L}^{\mathrm{hmg}}\right|_{\mathcal{S}^{\mathrm{hmg}}}$ is uniformly semi-normalized with respect to the family $\mathcal{N}_{\text {lin }}$. We call this kind of semi-normalization, which is characteristic for classes of homogeneous linear systems of differential equations, uniform seminormalization with respect to linear superposition of solutions. By Theorem 2, for each $\theta \in \mathcal{S}^{\mathrm{hmg}}$ the group $G_{\theta 0}$ splits over $G_{\theta 0}^{\text {lin }}$, and $G_{\theta 0}=G_{\theta 0}^{\text {ess }} \ltimes G_{\theta 0}^{\text {lin }}$, where $G_{\theta 0}^{\text {ess }}=\left.G_{\mathrm{hmg}}^{\sim}\right|_{(x, u)} \cap G_{\theta 0}$. By Theorem 3, the splitting of the point symmetry group induces a splitting of the corresponding maximal Lie invariance algebra: $\mathfrak{g}_{\theta 0}=\mathfrak{g}_{\theta 0}^{\text {ess }} \oplus \mathfrak{g}_{\theta 0}^{\text {lin }}$, where $\mathfrak{g}_{\theta 0}^{\text {ess }}$ is the essential Lie invariance algebra of $\mathcal{L}_{\theta 0}, \mathfrak{g}_{\theta 0}^{\text {ess }}=\left.\mathfrak{g}_{\mathrm{hmg}}^{\sim}\right|_{(x, u)} \cap \mathfrak{g}_{\theta 0}$, and the ideal $\mathfrak{g}_{\theta 0}^{\text {lin }}$, being the trivial part of $\mathfrak{g}_{\theta 0}$, consists of vector fields generating one-parameter symmetry groups of linear superposition of solutions. Thus, the group classification problem for the class $\left.\mathcal{L}^{\mathrm{hmg}}\right|_{\mathcal{S}^{\mathrm{hmg}}}$ reduces to the classification of appropriate subalgebras of the equivalence algebra $\mathfrak{g}_{\mathrm{hmg}}^{\sim}$ of this class. The qualification "appropriate" means that the restrictions of these subalgebras to the space of $(x, u)$ are essential Lie invariance algebras of systems from $\left.\mathcal{L}^{\mathrm{hmg}}\right|_{\mathcal{S}^{\mathrm{hmg}}}$.

For a class $\left.\mathcal{L}^{\mathrm{hmg}}\right|_{\mathcal{S}^{\mathrm{hmg}} \text { of linear homogeneous systems of differential equations that is uniformly }}$ semi-normalized with respect to the linear superposition of solutions, it is not necessary to start by considering the associated normalized superclass $\left.\mathcal{L}^{\text {inh }}\right|_{\mathcal{S}^{\text {inh }}}$ of generally inhomogeneous linear systems. The class $\left.\mathcal{L}^{\mathrm{hmg}}\right|_{\mathcal{S}^{\text {hmg }}}$ itself can be the starting point of the analysis. In order to get directly its uniform semi-normalization, we need to suppose the following properties of $\left.\mathcal{L}^{\mathrm{hmg}}\right|_{\mathcal{S}^{\mathrm{hmg}}}$, which are counterparts of the above properties of $\left.\mathcal{L}^{\mathrm{hmg}}\right|_{\mathcal{S}^{\mathrm{hmg}}}$ :

- Each system from $\left.\mathcal{L}^{\mathrm{hmg}}\right|_{\mathcal{S}^{\mathrm{hmg}}}$ is locally solvable.

- The zero function is the only common solution of systems from $\left.\mathcal{L}^{\mathrm{hmg}}\right|_{\mathcal{S}^{\mathrm{hmg}}}$.

- For any admissible transformation $\left(\theta^{1}, \theta^{2}, \varphi\right) \in \mathcal{G}_{\mathrm{hmg}}^{\sim}$, its transformational part $\varphi$ is of the form $\tilde{x}_{j}=X^{j}(x), \tilde{u}^{a}=M^{a b}(x)\left(u^{b}+h^{b}(x)\right)$, where $h=\left(h^{1}, \ldots, h^{m}\right)$ is a solution of $\mathcal{L}_{\theta^{1} 0}, \operatorname{det}\left(X_{x_{j^{\prime}}}^{j}\right) \neq 0$ and $\operatorname{det}\left(M^{a b}\right) \neq 0$. The functions $X^{j}$ 's and $M^{a b}$ 's may satisfy only additional constraints that do not depend on both $\theta^{1}$ and $\theta^{2}$.

The class (1) of linear Schrödinger equations fits very well into the framework which we use in the present paper to solve the group classification problem for this class.

Remark 4. There exist classes of homogeneous linear systems of differential equations that are uniformly semi-normalized with respect to symmetry-subgroup families different from the corresponding families of subgroups of linear superposition transformations. See Corollary [30 below. 
Remark 5. A technique similar to factoring out uniform Lie invariance algebras can be applied to kernel invariance algebras in the course of group classification of some normalized classes using the algebraic method. It is well known that for any class of differential equations $\left.\mathcal{L}\right|_{\mathcal{S}}$ the kernel $G^{\cap}$ of the maximal point symmetry groups $G_{\theta}$ 's of systems $\mathcal{L}_{\theta}$ 's from $\left.\mathcal{L}\right|_{\mathcal{S}}$ is a normal subgroup of the restriction $\left.G^{\sim}\right|_{(x, u)}$ of the (usual) equivalence group $G^{\sim}$ of $\left.\mathcal{L}\right|_{\mathcal{S}}$ to the space with local coordinates $(x, u),\left.G^{\cap} \triangleleft G^{\sim}\right|_{(x, u)}$. Analogues of this result can be found in the references [5. Proposition 3], [22, p. 52, Proposition 3.3.9], 35] and [34, Section II.6.5]. Furthermore, if the class $\left.\mathcal{L}\right|_{\mathcal{S}}$ is normalized, then the kernel $G^{\cap}$ is a normal subgroup of each $G_{\theta}, G^{\cap} \triangleleft G_{\theta}, \theta \in \mathcal{S}$ [5. Corollary 2]. However, the groups $\left.G^{\sim}\right|_{(x, u)}$ and $G_{\theta}$ 's do not in general split over $G^{\cap}$ even if the class $\left.\mathcal{L}\right|_{\mathcal{S}}$ is normalized. Similar assertions for the associated algebras are also true. See, in particular, Remark 9 and the subsequent subalgebra classification in [6] for a physically relevant example. Thus, the splitting of $G^{\sim}$ over $G^{\cap}$ does not follow from the normalization of $\left.\mathcal{L}\right|_{\mathcal{S}}$ and is an additional requirement for the kernel invariance algebra $\mathfrak{g}^{\cap}$ to be factored out when the group classification of $\left.\mathcal{L}\right|_{\mathcal{S}}$ is carried out.

\section{Equivalence groupoid}

In this section we find the equivalence groupoid $\mathcal{G}^{\sim}$ and the complete equivalence group $G^{\sim}$ of the class (11) in finite form (that is, not using the infinitesimal method). In the following $\mathcal{L}_{V}$ denotes the Schrödinger equation from the class (10) with potential $V=V(t, x)$. We look for all (locally) invertible point transformations of the form

$$
\tilde{t}=T\left(t, x, \psi, \psi^{*}\right), \quad \tilde{x}=X\left(t, x, \psi, \psi^{*}\right), \quad \tilde{\psi}=\Psi\left(t, x, \psi, \psi^{*}\right), \quad \tilde{\psi}^{*}=\Psi^{*}\left(t, x, \psi, \psi^{*}\right)
$$

(that is, $d T \wedge d X \wedge d \Psi \wedge d \Psi^{*} \neq 0$ ) that map a fixed equation $\mathcal{L}_{V}$ from the class (11) to an equation $\mathcal{L}_{\tilde{V}}: i \tilde{\psi}_{\tilde{t}}+\tilde{\psi}_{\tilde{x} \tilde{x}}+\tilde{V} \tilde{\psi}=0$ of the same class.

In the rest of this paper, we use the following notation: for any given complex number $\beta$

$$
\hat{\beta}=\beta \quad \text { if } \quad T_{t}>0 \quad \text { and } \quad \hat{\beta}=\beta^{*} \quad \text { if } \quad T_{t}<0 .
$$

Theorem 6. The equivalence groupoid $\mathcal{G}^{\sim}$ of the class (11) consists of triples of the form $(V, \tilde{V}, \varphi)$, where $\varphi$ is a point transformation acting on the space with local coordinates $(t, x, \psi)$ and given by

$$
\begin{aligned}
& \tilde{t}=T, \quad \tilde{x}=\varepsilon\left|T_{t}\right|^{1 / 2} x+X^{0}, \\
& \tilde{\psi}=\exp \left(\frac{i}{8} \frac{T_{t t}}{\left|T_{t}\right|} x^{2}+\frac{i}{2} \frac{\varepsilon \varepsilon^{\prime} X_{t}^{0}}{\left|T_{t}\right|^{1 / 2}} x+i \Sigma+\Upsilon\right)(\hat{\psi}+\hat{\Phi}),
\end{aligned}
$$

$V$ is an arbitrary potential and the transformed potential $\tilde{V}$ is related to $V$ by the equation

$$
\tilde{V}=\frac{\hat{V}}{\left|T_{t}\right|}+\frac{2 T_{t t t} T_{t}-3 T_{t t}^{2}}{16 \varepsilon^{\prime} T_{t}^{3}} x^{2}+\frac{\varepsilon \varepsilon^{\prime}}{2\left|T_{t}\right|^{1 / 2}}\left(\frac{X_{t}^{0}}{T_{t}}\right)_{t} x-\frac{i T_{t t}+\left(X_{t}^{0}\right)^{2}}{4 T_{t}^{2}}+\frac{\Sigma_{t}-i \Upsilon_{t}}{T_{t}},
$$

$T=T(t), X^{0}=X^{0}(t), \Sigma=\Sigma(t)$ and $\Upsilon=\Upsilon(t)$ are arbitrary smooth real-valued functions of $t$ with $T_{t} \neq 0$ and $\Phi=\Phi(t, x)$ denotes an arbitrary solution of the initial equation. $\varepsilon= \pm 1$ and $\varepsilon^{\prime}=\operatorname{sgn} T_{t}$.

Proof. The class (1) is a subclass of the class of generalized Schrödinger equations of the form $i \psi_{t}+\psi_{x x}+F=0$, where $\psi$ is a complex dependent variable of two real independent variables $t$ and $x$ and $F=F\left(t, x, \psi, \psi^{*}, \psi_{x}, \psi_{x}^{*}\right)$ is an arbitrary smooth complex-valued function of its arguments. This superclass is normalized, see [39], where it was also shown that any admissible transformation of the superclass satisfies the conditions

$$
T_{x}=T_{\psi}=T_{\psi^{*}}=0, \quad X_{\psi}=X_{\psi^{*}}=0, \quad X_{x}^{2}=\left|T_{t}\right|, \quad \Psi_{\hat{\psi}^{*}}=0 .
$$


Hence the same is true for the class (11). The equations (4) give us

$$
T=T(t), \quad X=\varepsilon\left|T_{t}\right|^{1 / 2} x+X^{0}(t), \quad \Psi=\Psi(t, x, \hat{\psi}),
$$

where $T$ and $X^{0}$ are arbitrary smooth real-valued functions of $t$ and $\Psi$ is an arbitrary smooth complex-valued function of its arguments. Then the invertibility of the transformation gives $T_{t} \neq 0$ and $\Psi_{\hat{\psi}} \neq 0$. Using the chain rule, we take total derivatives of the equation $\tilde{\psi}(\tilde{t}, \tilde{x})=$ $\Psi(t, x, \hat{\psi})$ with respect to $t$ and $x$, with $\tilde{t}=T$ and $\tilde{x}=X$ and we find the following expressions for the transformed derivatives:

$$
\begin{aligned}
& \tilde{\psi}_{\tilde{x}}=\frac{1}{X_{x}}\left(\Psi_{x}+\Psi_{\hat{\psi}} \hat{\psi}_{x}\right), \quad \tilde{\psi}_{\tilde{t}}=\frac{1}{T_{t}}\left(\Psi_{t}+\Psi_{\hat{\psi}} \hat{\psi}_{t}\right)-\frac{X_{t}}{T_{t} X_{x}}\left(\Psi_{x}+\Psi_{\hat{\psi}} \hat{\psi}_{x}\right), \\
& \tilde{\psi}_{\tilde{x} \tilde{x}}=\frac{1}{X_{x}^{2}}\left(\Psi_{x x}+2 \Psi_{x \hat{\psi}} \hat{\psi}_{x}+\Psi_{\hat{\psi} \hat{\psi}} \hat{\psi}_{x}^{2}+\Psi_{\hat{\psi}} \hat{\psi}_{x x}\right) .
\end{aligned}
$$

We substitute these expressions into the equation $\mathcal{L}_{\tilde{V}}$, and then take into account that $\psi$ satisfies the equation (11) so that $\hat{\psi}_{x x}=-\varepsilon^{\prime} i \hat{\psi}_{t}-\hat{V} \hat{\psi}$. We then split with respect to $\hat{\psi}_{t}$ and $\hat{\psi}_{x}$, which yields

$$
\begin{aligned}
& \Psi_{\hat{\psi} \hat{\psi}}=0, \quad \Psi_{x \hat{\psi}}=\frac{i}{2} \frac{X_{x}}{T_{t}} X_{t} \Psi_{\hat{\psi}}, \\
& \varepsilon^{\prime} i \Psi_{t}+\Psi_{x x}-\varepsilon^{\prime} i \frac{X_{t}}{X_{x}} \Psi_{x}+\left|T_{t}\right| \tilde{V} \Psi-\hat{V} \Psi_{\hat{\psi}} \hat{\psi}=0 .
\end{aligned}
$$

The general solution of the first equation in (5) is $\Psi=\Psi^{1}(t, x) \hat{\psi}+\Psi^{0}(t, x)$, where $\Psi^{0}$ and $\Psi^{1}$ are smooth complex-valued functions of $t$ and $x$. The second equation in (5) then reduces to a linear ordinary differential equation with respect to $\Psi^{1}$ with the independent variable $x$, and the variable $t$ plays the role of a parameter. Integrating this equation gives the following expression for $\Psi^{1}$ :

$$
\Psi^{1}=\exp \left(\frac{i}{8} \frac{T_{t t}}{\left|T_{t}\right|} x^{2}+\frac{i}{2} \frac{\varepsilon \varepsilon^{\prime} X_{t}^{0}}{\left|T_{t}\right|^{1 / 2}} x+i \Sigma(t)+\Upsilon(t)\right),
$$

where $\Sigma$ and $\Upsilon$ are arbitrary smooth real-valued functions of $t$. We substitute the expression for $\Psi$ into the equation (6) and then split this equation with respect to $\hat{\psi}$ and this then gives us the equation

$$
\tilde{V}=\frac{\hat{V}}{\left|T_{t}\right|}-\frac{1}{\left|T_{t}\right|} \frac{\Psi_{x x}^{1}}{\Psi^{1}}-\frac{i}{\left|T_{t}\right| \Psi^{1}}\left(\Psi_{t}^{1}-\frac{X_{t}}{X_{x}} \Psi_{x}^{1}\right) .
$$

which represents the component of the transformation (3) for $V$. We introduce the function $\Phi=\hat{\Psi}^{0} / \hat{\Psi}^{1}$, i.e., $\Psi^{0}=\Psi^{1} \hat{\Phi}$. The terms in (6) not containing $\hat{\psi}$ give an equation in $\Psi^{0}$, which is equivalent to the initial linear Schrödinger equation in terms of $\Phi$.

Corollary 7. A (1+1)-dimensional linear Schrödinger equation of the form (11) is equivalent to the free linear Schrödinger equation with respect to a point transformation if and only if $V=\gamma^{2}(t) x^{2}+\gamma^{1}(t) x+\gamma^{0}(t)+i \tilde{\gamma}^{0}(t)$ for some real-valued functions $\gamma^{2}, \gamma^{1}, \gamma^{0}$ and $\tilde{\gamma}^{0}$ of $t$.

Corollary 8. The usual equivalence group $G^{\sim}$ of the class (1) consists of point transformations of the form (3) with $\Phi=0$.

Proof. Each transformation from $G^{\sim}$ generates a family of admissible transformations for the class (11) and hence is of the form (3). As a usual equivalence group, the group $G^{\sim}$ merely consists of point transformations in the variables $(t, x, \psi)$ and the arbitrary element $V$ that can be applied to each equation from the class (11) and whose components for the variables are independent of $V$. The only transformations of the form (3) that satisfy these requirements are those for which $\Phi$ runs through the set of common solutions of all equations from the class (11). $\Phi=0$ is the only common solution. 
Remark 9. Consider the natural projection $\pi$ of the joint space of the variables and the arbitrary element $V$ on the space of the variables only. For each transformation $\mathcal{T}$ from the equivalence group $G^{\sim}$ its components for the variables do not depend on $V$ and are uniquely extended to $V$. These components define a transformation $\varphi$, which can be taken to be the pushforward of $\mathcal{T}$ by the projection $\pi, \varphi=\pi_{*} \mathcal{T}$. Therefore, there is a one-to-one correspondence between the equivalence group $G^{\sim}$ and the group $\pi_{*} G^{\sim}$ consisting of the projected equivalence transformations, $G^{\sim} \ni \mathcal{T} \mapsto \pi_{*} \mathcal{T} \in \pi_{*} G^{\sim}$.

Remark 10. The identity component of the equivalence group $G^{\sim}$ consists of transformations of the form (3) with $T_{t}>0$ and $\varepsilon=1$. This group also contains two discrete transformations that are involutions: the space reflection $\tilde{t}=t, \tilde{x}=-x, \tilde{\psi}=\psi, \tilde{V}=V$ and the Wigner time reflection $\tilde{t}=-t, \tilde{x}=x, \tilde{\psi}=\psi^{*}, \tilde{V}=V^{*}$, which are independent up to composition with each other and with continuous transformations. These continuous and discrete transformations generate the entire equivalence group $G^{\sim}$.

Corollary 11. The equivalence algebra of the class (11) is the algebra

$$
\mathfrak{g}^{\sim}=\langle\hat{D}(\tau), \hat{G}(\chi), \hat{M}(\sigma), \hat{I}(\rho)\rangle
$$

where $\tau, \chi, \sigma$ and $\rho$ run through the set of smooth real-valued functions of $t$. The vector fields that span $\mathfrak{g}^{\sim}$ are given by

$$
\begin{aligned}
\hat{D}(\tau)= & \tau \partial_{t}+\frac{1}{2} \tau_{t} x \partial_{x}+\frac{i}{8} \tau_{t t} x^{2}\left(\psi \partial_{\psi}-\psi^{*} \partial_{\psi^{*}}\right) \\
& -\left(\tau_{t} V-\frac{1}{8} \tau_{t t t} x^{2}-i \frac{\tau_{t t}}{4}\right) \partial_{V}-\left(\tau_{t} V^{*}-\frac{1}{8} \tau_{t t t} x^{2}+i \frac{\tau_{t t}}{4}\right) \partial_{V^{*}}, \\
\hat{G}(\chi)= & \chi \partial_{x}+\frac{i}{2} \chi_{t} x\left(\psi \partial_{\psi}-\psi^{*} \partial_{\psi^{*}}\right)+\frac{\chi_{t t}}{2} x\left(\partial_{V}+\partial_{V^{*}}\right), \\
\hat{M}(\sigma)= & i \sigma\left(\psi \partial_{\psi}-\psi^{*} \partial_{\psi^{*}}\right)+\sigma_{t}\left(\partial_{V}+\partial_{V^{*}}\right), \\
\hat{I}(\rho)= & \rho\left(\psi \partial_{\psi}+\psi^{*} \partial_{\psi^{*}}\right)-i \rho_{t}\left(\partial_{V}+\partial_{V^{*}}\right) .
\end{aligned}
$$

Proof. The equivalence algebra $\mathfrak{g}^{\sim}$ can be computed using the infinitesimal Lie method in a way similar to that for finding the Lie invariance algebra of a single system of differential equations [34. However, we can avoid these calculations by noting that we have already constructed the complete point equivalence group $G^{\sim}$. The algebra $\mathfrak{g}^{\sim}$ is just the set of infinitesimal generators of one-parameter subgroups of the group $G^{\sim}$. In order to find all such generators, in the transformation form (3) we set $\Phi=0$ (to single out equivalence transformations), $\varepsilon=1$ and $T_{t}>0$, i.e., $\varepsilon^{\prime}=1$ (to restrict to the continuous component of the identity transformation in $G^{\sim}$ ), and represent the parameter function $\Sigma$ in the form $\Sigma=\frac{1}{4} X^{0} X_{t}^{0}+\bar{\Sigma}$ with some function $\bar{\Sigma}$ of $t$ (to make the group parameterization more consistent with the one-parameter subgroup structure of $G^{\sim}$ ). Then we successively take one of the parameter-functions $T, X^{0}$, $\bar{\Sigma}$ and $\Upsilon$ to depend on a continuous subgroup parameter $\delta$, set the other parameter-functions to their trivial values, which are $t$ for $T$ and zeroes for $X^{0}, \bar{\Sigma}$ and $\Upsilon$, differentiate the transformation components with respect to $\delta$ and evaluate the result at $\delta=0$. The corresponding infinitesimal generator is the vector field $\tau \partial_{t}+\xi \partial_{x}+\eta \partial_{\psi}+\eta^{*} \partial_{\psi^{*}}+\theta \partial_{V}+\theta^{*} \partial_{V^{*}}$, where

$$
\tau=\left.\frac{\mathrm{d} \tilde{t}}{\mathrm{~d} \delta}\right|_{\delta=0}, \quad \xi=\left.\frac{\mathrm{d} \tilde{x}}{\mathrm{~d} \delta}\right|_{\delta=0}, \quad \eta=\left.\frac{\mathrm{d} \tilde{\psi}}{\mathrm{d} \delta}\right|_{\delta=0}, \quad \theta=\left.\frac{\mathrm{d} \tilde{V}}{\mathrm{~d} \delta}\right|_{\delta=0} .
$$

The above procedure gives the vector fields $\hat{D}(\tau), \hat{G}(\chi), \hat{M}(\sigma)$ and $\hat{I}(\rho)$ for the parameterfunctions $T, X^{0}, \bar{\Sigma}$ and $\Upsilon$, respectively. 
Consider the point symmetry group $G_{V}$ of an equation $\mathcal{L}_{V}$ from the class (11). Each element $\varphi$ of $G_{V}$ generates an admissible point transformation in the class (11) with the same initial and target arbitrary element $V$. Therefore, the components of $\varphi$ necessarily have the form given in (3a) - (3b), and the parameter-functions satisfy the equation (3c) with $\tilde{V}(\tilde{t}, \tilde{x})=V(\tilde{t}, \tilde{x})$. The symmetry transformations defined by linear superposition of solutions to the equation $\mathcal{L}_{V}$ are of the form given in (3a) - (3b) with $T=t$ and $X^{0}=\Sigma=\Upsilon=0$. They constitute a normal subgroup $G_{V}^{\text {lin }}$ of the group $G_{V}$, which can be assumed to be the trivial part of $G_{V}$. The factor group $G_{V} / G_{V}^{\mathrm{lin}}$ is isomorphic to the subgroup $G_{V}^{\text {ess }}$ of $G_{V}$ that is singled out from $G_{V}$ by the constraint $\Phi=0$ and will be considered as the only essential part of $G_{V}$.

Corollary 12. The essential point symmetry group $G_{V}^{\mathrm{ess}}$ of any equation $\mathcal{L}_{V}$ from the class (11) is contained in the projection $\pi_{*} G^{\sim}$ of the equivalence group $G^{\sim}$ to the space with local coordinates $\left(t, x, \psi, \psi^{*}\right)$.

Corollary 13. The class (11) is uniformly semi-normalized with respect to linear superposition of solutions.

Proof. We need to show that any admissible transformation in the class (11) is the composition of a specific symmetry transformation of the initial equation and a transformation from $G^{\sim}$. We consider two fixed similar equations $\mathcal{L}_{V}$ and $\mathcal{L}_{\tilde{V}}$ in the class (11) and let $\varphi$ be a point transformation connecting these equations. Then $\varphi$ is of the form (3a) $-(3 \mathrm{~b})$, and the potentials $V$ and $\tilde{V}$ are related by (3c). The point transformation $\varphi^{1}$ given by $\tilde{t}=t, \tilde{x}=x, \tilde{\psi}=\psi+\Phi$ with the same function $\Phi$ as in $\varphi$ is a symmetry transformation of the initial equation, which is related to the linear superposition principle. We choose the transformation $\varphi^{2}$ to be of the same form (3a) $-(3 \mathrm{~b})$ but with $\Phi=0$. By (3c), its extension to the arbitrary element belongs to the group $G^{\sim}$. The transformation $\varphi$ is the composition of $\varphi^{1}$ and $\varphi^{2}$.

It is obvious that the transformation $\varphi$ maps the subgroup of linear superposition transformations of the equation $\mathcal{L}_{V}$ onto that of the equation $\mathcal{L}_{\tilde{V}}$.

\section{Analysis of determining equations for Lie symmetries}

We derive the determining equations for elements from the maximal Lie invariance algebra $\mathfrak{g}_{V}$ of an equation $\mathcal{L}_{V}$ from the class (10) with potential $V=V(t, x)$. The general form of a vector field $Q$ in the space with local coordinates $\left(t, x, \psi, \psi^{*}\right)$ is $Q=\tau \partial_{t}+\xi \partial_{x}+\eta \partial_{\psi}+\eta^{*} \partial_{\psi^{*}}$, where the components of $Q$ are smooth functions of $\left(t, x, \psi, \psi^{*}\right)$. The vector field $Q$ belongs to the algebra $\mathfrak{g}_{V}$ if and only if it satisfies the infinitesimal invariance criterion for the equation $\mathcal{L}_{V}$, which gives

$$
i \eta^{t}+\eta^{x x}+\tau V_{t} \psi+\xi V_{x} \psi+V \eta=0
$$

for all solutions of $\mathcal{L}_{V}$. Here

$$
\begin{aligned}
& \eta^{t}=D_{t}\left(\eta-\tau \psi_{t}-\xi \psi_{x}\right)+\tau \psi_{t t}+\xi \psi_{t x}, \\
& \eta^{x x}=D_{x}^{2}\left(\eta-\tau \psi_{t}-\xi \psi_{x}\right)+\tau \psi_{t x x}+\xi \psi_{x x x},
\end{aligned}
$$

$D_{t}$ and $D_{x}$ denote the total derivative operators with respect to $t$ and $x$, respectively. After substituting $\psi_{x x}=-i \psi_{t}-V \psi$ and $\psi_{x x}^{*}=i \psi_{t}^{*}-V^{*} \psi^{*}$ into (7) and splitting with respect to the other derivatives of $\psi$ and $\psi^{*}$ that occur, we obtain a linear overdetermined system of determining equations for the components of $Q$,

$$
\begin{aligned}
& \tau_{\psi}=\tau_{\psi^{*}}=0, \quad \tau_{x}=0, \quad \xi_{\psi}=\xi_{\psi^{*}}=0, \quad \tau_{t}=2 \xi_{x}, \quad \eta_{\psi^{*}}=\eta_{\psi \psi}=0, \quad 2 \eta_{x \psi}=i \xi_{t}, \\
& i \eta_{t}+\eta_{x x}+\tau V_{t} \psi+\xi V_{x} \psi+V \eta-\left(\eta_{\psi}-\tau_{t}\right) V \psi=0 .
\end{aligned}
$$


We solve the determining equations in the first line to obtain

$$
\tau=\tau(t), \quad \xi=\frac{1}{2} \tau_{t} x+\chi(t), \quad \eta=\left(\frac{i}{8} \tau_{t t} x^{2}+\frac{i}{2} \chi_{t} x+\rho(t)+i \sigma(t)\right) \psi+\eta^{0}(t, x),
$$

where $\tau, \chi, \rho$ and $\sigma$ are smooth real-valued functions of $t$, and $\eta^{0}$ is a complex-valued function of $t$ and $x$. Then splitting the last determining equation with respect to $\psi$, we derive two equations:

$$
\begin{aligned}
& i \eta_{t}^{0}+\eta_{x x}^{0}+\eta^{0} V=0, \\
& \tau V_{t}+\left(\frac{1}{2} \tau_{t} x+\chi\right) V_{x}+\tau_{t} V=\frac{1}{8} \tau_{t t t} x^{2}+\frac{1}{2} \chi_{t t} x+\sigma_{t}-i \rho_{t}-\frac{i}{4} \tau_{t t} .
\end{aligned}
$$

Although both these equations contain the potential $V$, the first equation just means that the parameter-function $\eta^{0}$ satisfies the equation $\mathcal{L}_{V}$, which does not affect the structure of the algebra $\mathfrak{g}_{V}$ when the potential $V$ varies. This is why we only consider the second equation as the real classifying condition for Lie symmetry operators of equations from the class (1).

We have thus proved the following result:

Theorem 14. The maximal Lie invariance algebra $\mathfrak{g}_{V}$ of the equation $\mathcal{L}_{V}$ from the class (1) consists of the vector fields of the form $Q=D(\tau)+G(\chi)+\sigma M+\rho I+Z\left(\eta^{0}\right)$, where

$$
\begin{aligned}
& D(\tau)=\tau \partial_{t}+\frac{1}{2} \tau_{t} x \partial_{x}+\frac{1}{8} \tau_{t t} x^{2} M, \quad G(\chi)=\chi \partial_{x}+\frac{1}{2} \chi_{t} x M, \\
& M=i \psi \partial_{\psi}-i \psi^{*} \partial_{\psi^{*}}, \quad I=\psi \partial_{\psi}+\psi^{*} \partial_{\psi^{*}}, \quad Z\left(\eta^{0}\right)=\eta^{0} \partial_{\psi}+\eta^{0 *} \partial_{\psi^{*}},
\end{aligned}
$$

the parameters $\tau, \chi, \rho, \sigma$ run through the set of real-valued smooth functions of $t$ satisfying the classifying condition (9), and $\eta^{0}$ runs through the solution set of the equation $\mathcal{L}_{V}$.

Note that Theorem 14 can be derived from Theorem 6 using the same technique as in Corollary 11. The algebra $\mathfrak{g}_{V}$ consists of infinitesimal generators of one-parameter subgroups of the point symmetry group $G_{V}$ of the equation $\mathcal{L}_{V}$. In considering one-parameter subgroups of $G_{V}$, we set $\varepsilon=1$ and $T_{t}>0$, i.e., $\varepsilon^{\prime}=1$ since one-parameter subgroups are contained in the identity component of $G_{V}$. We also represent $\Sigma$ in the form $\Sigma=\frac{1}{4} X^{0} X_{t}^{0}+\bar{\Sigma}$. We let the parameter functions $T, X^{0}, \bar{\Sigma}, \Upsilon$ and $\Phi$ properly depend on a continuous subgroup parameter $\delta$. Then we differentiate the equations (3) with respect to $\delta$ and evaluate the result at $\delta=0$. The corresponding infinitesimal generator is the vector field $Q=\tau \partial_{t}+\xi \partial_{x}+\eta \partial_{\psi}+\eta^{*} \partial_{\psi^{*}}$, where

$$
\tau=\left.\frac{\mathrm{d} \tilde{t}}{\mathrm{~d} \delta}\right|_{\delta=0}, \quad \xi=\left.\frac{\mathrm{d} \tilde{x}}{\mathrm{~d} \delta}\right|_{\delta=0}, \quad \eta=\left.\frac{\mathrm{d} \tilde{\psi}}{\mathrm{d} \delta}\right|_{\delta=0},
$$

and hence $Q$ has the form given in Theorem 14. The classifying condition (9) is derived from the equation (3c) with $\tilde{V}(\tilde{t}, \tilde{x})=V(\tilde{t}, \tilde{x})$.

In order to find the kernel invariance algebra $\mathfrak{g}^{\cap}$ of the class (1), $\mathfrak{g}^{\cap}:=\bigcap_{V} \mathfrak{g}_{V}$, we vary the potential $V$ and then split the equations (8) and (9) with respect to $V$ and its derivatives. This gives us the equations $\tau=\chi=0, \eta^{0}=0$ and $\rho_{t}=\sigma_{t}=0$.

Proposition 15. The kernel invariance algebra of the class (11) is the algebra $\mathfrak{g}^{\cap}=\langle M, I\rangle$.

Consider the linear span of all vector fields from Theorem 14 when $V$ varies given by

$$
\mathfrak{g}_{\langle\rangle}:=\langle D(\tau), G(\chi), \sigma M, \rho I, Z(\zeta)\rangle=\sum_{V} \mathfrak{g}_{V} .
$$

Here and in the following the parameters $\tau, \chi, \sigma$ and $\rho$ run through the set of real-valued smooth functions of $t, \zeta$ runs through the set of complex-valued smooth functions of $(t, x)$ and $\eta^{0}$ runs 
through the solution set of the equation $\mathcal{L}_{V}$ when the potential $V$ is fixed. We have $\mathfrak{g}_{\langle\rangle}=\sum_{V} \mathfrak{g}_{V}$ since each vector field $Q$ from $\mathfrak{g}_{\langle\rangle}$with nonvanishing $\tau$ or $\chi$ or with jointly vanishing $\tau, \chi, \sigma$ and $\rho$ necessarily belongs to $\mathfrak{g}_{V}$ for some $V$. The nonzero commutation relations between vector fields spanning $\mathfrak{g}_{\langle>}$are

$$
\begin{aligned}
& {\left[D\left(\tau^{1}\right), D\left(\tau^{2}\right)\right]=D\left(\tau^{1} \tau_{t}^{2}-\tau^{2} \tau_{t}^{1}\right), \quad[D(\tau), G(\chi)]=G\left(\tau \chi_{t}-\frac{1}{2} \tau_{t} \chi\right)} \\
& {[D(\tau), \sigma M]=\tau \sigma_{t} M, \quad[D(\tau), \rho I]=\tau \rho_{t} I} \\
& {[D(\tau), Z(\zeta)]=Z\left(\tau \zeta_{t}+\frac{1}{2} \tau_{t} x \zeta_{x}-\frac{i}{8} \tau_{t t} x^{2} \zeta\right),} \\
& {\left[G\left(\chi^{1}\right), G\left(\chi^{2}\right)\right]=\left(\chi^{1} \chi_{t}^{2}-\chi^{2} \chi_{t}^{1}\right) M, \quad[G(\chi), Z(\zeta)]=Z\left(\chi \zeta_{x}-\frac{i}{2} \chi_{t} x \zeta\right),} \\
& {[\sigma M, Z(\zeta)]=Z(-i \sigma \zeta), \quad[\rho I, Z(\zeta)]=Z(-\rho \zeta) .}
\end{aligned}
$$

The commutation relations between elements of $\mathfrak{g}_{\langle\rangle}$show that $\mathfrak{g}_{\langle\rangle}$itself is a Lie algebra. It is convenient to represent $\mathfrak{g}_{\langle\backslash}$ as a semi-direct sum,

$$
\mathfrak{g}_{\langle\rangle}=\mathfrak{g}_{\langle\rangle}^{\text {ess }} \in \mathfrak{g}_{\langle\rangle}^{\text {lin }}, \quad \text { where } \quad \mathfrak{g}_{\langle\rangle}^{\text {ess }}:=\langle D(\tau), G(\chi), \sigma M, \rho I\rangle \quad \text { and } \quad \mathfrak{g}_{\langle\rangle}^{\operatorname{lin}}:=\langle Z(\zeta)\rangle
$$

are a subalgebra and an abelian ideal of $\mathfrak{g}_{\langle\rangle}$, respectively. Note that the kernel invariance algebra $\mathfrak{g}^{\cap}$ is an ideal of $\mathfrak{g}_{\langle\rangle}^{\text {ess }}$ and of $\mathfrak{g}_{\langle\rangle}$. The above representation of $\mathfrak{g}_{\langle\rangle}$induces a similar representation for each $\mathfrak{g}_{V}$,

$$
\mathfrak{g}_{V}=\mathfrak{g}_{V}^{\text {ess }} \oplus \mathfrak{g}_{V}^{\text {lin }}, \quad \text { where } \quad \mathfrak{g}_{V}^{\text {ess }}:=\mathfrak{g}_{V} \cap \mathfrak{g}_{\langle\rangle}^{\text {ess }} \quad \text { and } \quad \mathfrak{g}_{V}^{\operatorname{lin}}:=\mathfrak{g}_{V} \cap \mathfrak{g}_{\langle\rangle}^{\operatorname{lin}}=\left\langle Z\left(\eta^{0}\right), \eta^{0} \in \mathcal{L}_{V}\right\rangle
$$

are a finite-dimensional subalgebra (see Lemma 18 below) and an infinite-dimensional abelian ideal of $\mathfrak{g}_{V}$, respectively. We call $\mathfrak{g}_{V}^{\text {ess }}$ the essential Lie invariance algebra of the equation $\mathcal{L}_{V}$ for each $V$. The ideal $\mathfrak{g}_{V}^{\text {lin }}$ consists of vector fields associated with transformations of linear superposition and therefore it is a trivial part of $\mathfrak{g}_{V}$.

Definition 16. A subalgebra $\mathfrak{s}$ of $\mathfrak{g}_{\langle\backslash}^{\text {ess }}$ is called appropriate if there exists a potential $V$ such that $\mathfrak{s}=\mathfrak{g}_{V}^{\text {ess }}$.

The algebras $\mathfrak{g}_{\langle\rangle}^{\text {ess }}$ and $\mathfrak{g}^{\sim}$ are related to each other by $\mathfrak{g}_{\langle\rangle}^{\text {ess }}=\pi_{*} \mathfrak{g}^{\sim}$, where $\pi$ is the projection of the joint space of the variables and the arbitrary element on the space of the variables only. The mapping $\pi_{*}$ induced by $\pi$ is well defined on $\mathfrak{g}^{\sim}$ due to the structure of elements of $\mathfrak{g}^{\sim}$. Note that the vector fields $\hat{D}(\tau), \hat{G}(\chi), \hat{M}(\sigma), \hat{I}(\rho)$ spanning $\mathfrak{g}^{\sim}$ are mapped by $\pi_{*}$ to the vector fields $D(\tau)$, $G(\chi), \sigma M, \rho I$ spanning $\mathfrak{g}_{\langle\rangle}^{\text {ess }}$, respectively. The above relation is stronger than that implied by the specific semi-normalization of the class (1), $\mathfrak{g}_{\langle\rangle}^{\text {ess }} \subseteq \pi_{*} \mathfrak{g}^{\sim}$. Since the algebra $\mathfrak{g}_{\langle\rangle}^{\text {ess }}$ coincides with the set $\pi_{*} \mathfrak{g}^{\sim}$ of infinitesimal generators of one-parameter subgroups of the group $\pi_{*} G^{\sim}$, the structure of $\mathfrak{g}_{\langle\rangle}^{\text {ess }}$ is compatible with the action of $\pi_{*} G^{\sim}$ on this algebra. Moreover, both $\mathfrak{g}_{\langle\rangle}^{\text {ess }}$ and $\mathfrak{g}_{\langle>}^{\operatorname{lin}}$ are invariant with respect to the action of the group $\pi_{*} G^{\sim}$. This is why the action of $G^{\sim}$ on equations from the class (1) induces the well-defined action of $\pi_{*} G^{\sim}$ on the essential Lie invariance algebras of these equations, which are subalgebras of $\mathfrak{g}_{\langle\rangle}^{\text {ess }}$. The kernel $\mathfrak{g}^{\cap}$ is obviously an ideal in $\mathfrak{g}_{V}^{\text {ess }}$ for any $V$.

Collecting all the above arguments, we obtain the following assertion.

Proposition 17. The problem of group classification of (1+1)-dimensional linear Schrödinger equations reduces to the classification of appropriate subalgebras of the algebra $\mathfrak{g}_{\langle\rangle}^{\text {ess }}$ with respect to the equivalence relation generated by the action of $\pi_{*} G^{\sim}$.

Equivalently, we can classify the counterparts of appropriate subalgebras in $\mathfrak{g}^{\sim}$ up to $G^{\sim}$ equivalence and then project them to the space of variables [6, 13]. 


\section{Group classification}

To classify appropriate subalgebras of the algebra $\mathfrak{g}_{\langle\rangle}^{\text {ess }}$, we need to compute the action of transformations from the group $\pi_{*} G^{\sim}$ on vector fields from $\mathfrak{g}_{\langle\rangle}^{\text {ess }}$. For any transformation $\varphi \in \pi_{*} G^{\sim}$ and any vector field $Q \in \mathfrak{g}_{\langle\rangle}^{\text {ess }}$, the pushforward action of $\varphi$ on $Q$ is given by

$$
\tilde{Q}:=\varphi_{*} Q=Q(T) \partial_{\tilde{t}}+Q(X) \partial_{\tilde{x}}+Q(\Psi) \partial_{\tilde{\psi}}+Q\left(\Psi^{*}\right) \partial_{\tilde{\psi}^{*}},
$$

where in each component of $\tilde{Q}$ we substitute the expressions of the variables without tildes in terms of the "tilded" variables, $\left(t, x, \psi, \psi^{*}\right)=\varphi^{-1}\left(\tilde{t}, \tilde{x}, \tilde{\psi}, \tilde{\psi}^{*}\right)$, and $\varphi^{-1}$ denotes the inverse of $\varphi$.

For convenience, we introduce the following notation for elementary transformations from $\pi_{*} G^{\sim}$, which generate the entire group $\pi_{*} G^{\sim}: \mathcal{D}(T), \mathcal{G}\left(X^{0}\right), \mathcal{M}(\Sigma)$ and $\mathcal{I}(\Upsilon)$ respectively denote the transformations of the form (3a) - (3b) with $\Phi=0$ and $\varepsilon=1$, where the parameterfunctions $T, X^{0}, \Sigma$ and $\Upsilon$, successively excluding one of them, are set to the values corresponding to the identity transformation, which are $t$ for $T$ and zeroes for $X^{0}, \Sigma$ and $\Upsilon$. The nontrivial pushforward actions of elementary transformations from $\pi_{*} G^{\sim}$ on the vector fields spanning $\mathfrak{g}_{\langle\rangle}^{\text {ess }}$ are

$$
\begin{aligned}
& \mathcal{D}_{*}(T) D(\tau)=\tilde{D}\left(T_{t} \tau\right), \quad \mathcal{D}_{*}(T) G(\chi)=\tilde{G}\left(T_{t}^{1 / 2} \chi\right) \\
& \mathcal{D}_{*}(T)(\sigma M)=\sigma \tilde{M}, \quad \mathcal{D}_{*}(T)(\rho I)=\rho \tilde{I} \\
& \mathcal{G}_{*}\left(X^{0}\right) D(\tau)=\tilde{D}(\tau)+\tilde{G}\left(\tau X_{t}^{0}-\frac{1}{2} \tau_{t} X^{0}\right)+\left(\frac{1}{8} \tau_{t t}\left(X^{0}\right)^{2}-\frac{1}{4} \tau_{t} X^{0} X_{t}^{0}-\frac{1}{2} \tau X^{0} X_{t t}^{0}\right) \tilde{M} \\
& \mathcal{G}_{*}\left(X^{0}\right) G(\chi)=\tilde{G}(\chi)+\frac{1}{2}\left(\chi X_{t}^{0}-\chi_{t} X^{0}\right) \tilde{M} \\
& \mathcal{M}_{*}(\Sigma) D(\tau)=\tilde{D}(\tau)+\tau \Sigma_{t} \tilde{M}, \quad \mathcal{I}_{*}(\Upsilon) D(\tau)=\tilde{D}(\tau)+\tau \Upsilon_{t} \tilde{I}
\end{aligned}
$$

where in each pushforward by $\mathcal{D}_{*}(T)$ we should substitute the expression for $t$ given by inverting the relation $\tilde{t}=T(t) ; t=\tilde{t}$ for the other pushforwards. Tildes over vector fields mean that these vector fields are represented in the new variables.

Lemma 18. $\operatorname{dim} \mathfrak{g}_{V}^{\mathrm{ess}} \leqslant 7$ for any potential $V$.

Proof. Since we work within the local framework, we can assume that the equation $\mathcal{L}_{V}$ is considered on a domain of the form $\Omega_{0} \times \Omega_{1}$, where $\Omega_{0}$ and $\Omega_{1}$ are intervals on the $t$ - and $x$-axes, respectively. Then we successively evaluate the classifying condition (9) at three different points $x=x_{0}-\delta, x=x_{0}$ and $x=x_{0}+\delta$ from $\Omega_{1}$ for varying $t$. This gives

$$
\begin{aligned}
& \frac{1}{8} \tau_{t t t}\left(x_{0}-\delta\right)^{2}+\frac{1}{2} \chi_{t t}\left(x_{0}-\delta\right)-i \rho_{t}+\sigma_{t}-\frac{i}{4} \tau_{t t}=R_{1}, \\
& \frac{1}{8} \tau_{t t t} x_{0}^{2}+\frac{1}{2} \chi_{t t} x_{0}-i \rho_{t}+\sigma_{t}-\frac{i}{4} \tau_{t t}=R_{2}, \\
& \frac{1}{8} \tau_{t t t}\left(x_{0}+\delta\right)^{2}+\frac{1}{2} \chi_{t t}\left(x_{0}+\delta\right)-i \rho_{t}+\sigma_{t}-\frac{i}{4} \tau_{t t}=R_{3},
\end{aligned}
$$

where the right hand sides $R_{1}, R_{2}$ and $R_{3}$ are the results of substituting the above values of $x$ into $\tau V_{t}+\left(\frac{1}{2} \tau_{t} x+\chi\right) V_{x}+\tau_{t} V$. Combining the above equations and splitting them into real and imaginary parts, we obtain a canonical system of linear ordinary differential equations of the form

$$
\tau_{t t t}=\ldots, \quad \chi_{t t}=\ldots, \quad \rho_{t}=\ldots, \quad \sigma_{t}=\ldots
$$

to $\tau, \chi, \rho$ and $\sigma$. The qualification "canonical" means that the system is solved with respect to the highest-order derivatives. The right hand sides of all its equations are denoted by dots since their precise form is not important for our argument. It is obvious that the solution set of the above system is a linear space and parameterized by seven arbitrary constants. 
In order to classify appropriate subalgebras of $\mathfrak{g}_{\langle\supset}^{\text {ess }}$, for each subalgebra $\mathfrak{s}$ of $\mathfrak{g}_{\langle\rangle}^{\text {ess }}$ we introduce two integers

$$
k_{1}=k_{1}(\mathfrak{s}):=\operatorname{dim} \pi_{*}^{0} \mathfrak{s}, \quad k_{2}=k_{2}(\mathfrak{s}):=\operatorname{dim} \mathfrak{s} \cap\langle G(\chi), \sigma M, \rho I\rangle-2,
$$

where $\pi^{0}$ denotes the projection onto the space of the variable $t$ and $\pi_{*}^{0} \mathfrak{s} \subset \pi_{*}^{0} \mathfrak{g}_{\langle\rangle}^{\text {ess }}=\left\langle\tau \partial_{t}\right\rangle$. The values of $k_{1}$ and $k_{2}$ are invariant under the action of $\pi_{*} G^{\sim}$.

Lemma 19. $\pi_{*}^{0} \mathfrak{g}_{V}^{\text {ess }}$ is a Lie algebra for any potential $V$ and $k_{1}=\operatorname{dim} \pi_{*}^{0} \mathfrak{g}_{V}^{\text {ess }} \leqslant 3$. Further, $\pi_{*}^{0} \mathfrak{g}_{V}^{\text {ess }} \in\left\{0,\left\langle\partial_{t}\right\rangle,\left\langle\partial_{t}, t \partial_{t}\right\rangle,\left\langle\partial_{t}, t \partial_{t}, t^{2} \partial_{t}\right\rangle\right\} \bmod \pi_{*}^{0} G^{\sim}$.

Proof. To prove that $\pi_{*}^{0} \mathfrak{g}_{V}^{\text {ess }}$ is a Lie algebra we show that it is a linear subspace and closed under Lie bracket of vector fields. Given $\tau^{j} \partial_{t} \in \pi_{*}^{0} \mathfrak{g}_{V}^{\text {ess }}, j=1,2$, there exist $Q^{j} \in \mathfrak{g}_{V}^{\text {ess }}$ such that $\pi_{*}^{0} Q^{j}=\tau^{j} \partial_{t}$. Then for any real constants $c_{1}$ and $c_{2}$ we have $c_{1} Q^{1}+c_{2} Q^{2} \in \mathfrak{g}_{V}^{\text {ess }}$. Therefore, $c_{1} \tau^{1} \partial_{t}+c_{2} \tau^{2} \partial_{t}=\pi_{*}^{0}\left(c_{1} Q^{1}+c_{2} Q^{2}\right) \in \pi_{*}^{0} \mathfrak{g}_{V}^{\text {ess }}$. Next, $\left[\tau^{1} \partial_{t}, \tau^{2} \partial_{t}\right]=\left(\tau^{1} \tau_{t}^{2}-\tau^{2} \tau_{t}^{1}\right) \partial_{t}=\pi_{*}^{0}\left[Q^{1}, Q^{2}\right] \in$ $\pi_{*}^{0} \mathfrak{g}_{V}^{\text {ess }}$. Further, $\operatorname{dim} \pi_{*}^{0} \mathfrak{g}_{V}^{\text {ess }} \leqslant \operatorname{dim} \mathfrak{g}_{V}^{\text {ess }} \leqslant 7$.

Thus $\pi_{*}^{0} \mathfrak{g}_{V}^{\text {ess }}$ is a finite-dimensional subalgebra of the Lie algebra $\pi_{*}^{0} \mathfrak{g}_{\langle\rangle}^{\text {ess }}$ of vector fields on the real line. The group $\pi_{*}^{0} G^{\sim}$ coincides with the entire group of local diffeomorphisms of the real line and the rest of the lemma follows from Lie's theorem on finite-dimensional Lie algebras of vector fields on the real line.

Lemma 20. If a vector field $Q$ is of the form $Q=G(\chi)+\sigma M+\rho I$ with $\chi \neq 0$, then $Q=$ $G(1)+\tilde{\rho} I \bmod \pi_{*} G^{\sim}$ for another function $\tilde{\rho}$.

Proof. We successively push forward the vector field $Q$ by the transformations $\mathcal{G}\left(X^{0}\right)$ and $\mathcal{D}(T)$, where $X^{0}$ and $T$ are arbitrary fixed solutions of the ordinary differential equations $\chi X_{t}^{0}-\chi_{t} X^{0}=$ $2 \sigma$ and $T_{t}=1 / \chi^{2}$, respectively. This leads to a vector field of the same form, with $\chi=1$ and $\sigma=0$.

Lemma 21. If $G(1)+\rho^{1} I \in \mathfrak{g}_{V}^{\text {ess }}$, then also $G(t)+\rho^{2} I \in \mathfrak{g}_{V}^{\text {ess }}$ with $\rho^{2}=\int t \rho_{t}^{1} \mathrm{~d} t$.

Proof. The fact that $G(1)+\rho^{1} I \in \mathfrak{g}_{V}^{\text {ess }}$ means that the values $\tau=\sigma=0, \chi=1$ and $\rho=\rho^{1}$ satisfy the classifying condition (9) with the given potential $V$, which gives $V_{x}=-i \rho_{t}$. Then $t V_{x}=-i t \rho_{t}$ implies that the classifying condition (9) is also satisfied by $\tau=\sigma=0, \chi=t$ and $\rho^{2}=\int t \rho_{t}^{1} \mathrm{~d} t$.

Lemma 22. $\mathfrak{g}_{V}^{\mathrm{ess}} \cap\langle\sigma M, \rho I\rangle=\mathfrak{g}^{\cap}$ for any potential $V$.

Proof. We need to show that $\mathfrak{g}_{V}^{\text {ess }} \cap\langle\sigma M, \rho I\rangle \subset \mathfrak{g}^{\cap}$ and $\mathfrak{g}^{\cap} \subset \mathfrak{g}_{V}^{\text {ess }} \cap\langle\sigma M, \rho I\rangle$. The first inclusion follows from the classifying condition (9) for $\tau=\chi=0$, which implies $\sigma_{t}=\rho_{t}=0$. The second inclusion is obvious since the kernel invariance algebra $\mathfrak{g}^{\cap}$ is contained in $\mathfrak{g}_{V}^{\text {ess }}$ for any $V$.

Lemma 23. $k_{2}=\operatorname{dim} \mathfrak{g}_{V}^{\text {ess }} \cap\langle G(\chi), \sigma M, \rho I\rangle-2 \in\{0,2\}$ for any potential $V$.

Proof. Denote $\mathfrak{a}_{V}:=\mathfrak{g}_{V}^{\text {ess }} \cap\langle G(\chi), \sigma M, \rho I\rangle$.

If $\mathfrak{a}_{V} \subseteq\langle\sigma M, \rho I\rangle$, then $\mathfrak{a}_{V}=\mathfrak{g}_{V}^{\text {ess }} \cap\langle\sigma M, \rho I\rangle=\mathfrak{g}^{\cap}$, i.e., $k_{2}=\operatorname{dim} \mathfrak{a}_{V}-2=0$.

If $\mathfrak{a}_{V} \nsubseteq\langle\sigma M, \rho I\rangle$, then there exists $Q^{1} \in \mathfrak{a}_{V}$ such that $Q^{1} \notin\langle\sigma M, \rho I\rangle$. From Lemma 20, up to $\pi_{*} G^{\sim}$-equivalence we may assume that $Q^{1}$ is locally of the form $Q^{1}=G(1)+\rho^{1} I$. Then Lemma21]implies that $Q^{2}=G(t)+\rho^{2} I$ with $\rho^{2}=\int t \rho_{t}^{1} \mathrm{~d} t$ belongs to $\mathfrak{a}_{V}$. We also have $\mathfrak{a}_{V} \supset \mathfrak{g}^{\cap}$. It then follows that $\left\langle M, I, Q^{1}, Q^{2}\right\rangle \subseteq \mathfrak{a}_{V}$ and hence $\operatorname{dim} \mathfrak{a}_{V} \geqslant 4$. On the other hand, as follows from the proof of Lemma 18 under the constraint $\tau=0$, the classifying condition (9) implies in particular a canonical system of linear ordinary differential equations of the form

$$
\chi_{t t}=\ldots, \quad \rho_{t}=\ldots, \quad \sigma_{t}=\ldots
$$

in the parameter-functions $\chi, \rho$ and $\sigma$, whose solution space is four-dimensional. This means that $\operatorname{dim} \mathfrak{a}_{V} \leqslant 4$. Therefore, $k_{2}=\operatorname{dim} \mathfrak{a}_{V}-2=2$. 
Summarizing the above results, any appropriate subalgebra of $\mathfrak{g}_{\langle>}^{\text {ess }}$ is spanned by

- the basis vector fields $M$ and $I$ of the kernel $\mathfrak{g}^{\bigcap}$,

- $k_{1}$ vector fields $D\left(\tau^{j}\right)+G\left(\chi^{j}\right)+\sigma^{j} M+\rho^{j} I$, where $j=1, \ldots, k_{1}, k_{1} \leqslant 3$, and $\tau^{1}, \ldots, \tau^{k_{1}}$ are linearly independent,

- $k_{2}$ vector fields $G\left(\chi^{l}\right)+\sigma^{l} M+\rho^{l} I$ where $l=1, \ldots, k_{2}, k_{2} \in\{0,2\}$ and $\chi^{1}, \ldots, \chi^{k_{2}}$ are linearly independent.

Theorem 24. A complete list of $G^{\sim}$-inequivalent (and, therefore, $\mathcal{G}^{\sim}$-inequivalent) Lie symmetry extensions in the class (1) is exhausted by the cases collected in Table 1.

Table 1. Results of classification.

\begin{tabular}{|c|c|c|c|l|}
\hline no. & $k_{1}$ & $k_{2}$ & $V$ & \multicolumn{1}{|c|}{ Basis of $\mathfrak{g}_{V}^{\text {ess }}$} \\
\hline 1 & 0 & 0 & $V(t, x)$ & $M, I$ \\
2 & 0 & 2 & $i \gamma(t) x$ & $M, I, G(1)-\left(\int \gamma(t) \mathrm{d} t\right) I, G(t)-\left(\int t \gamma(t) \mathrm{d} t\right) I$ \\
3 & 1 & 0 & $V(x)$ & $M, I, D(1)$ \\
$4 \mathrm{a}$ & 1 & 2 & $\frac{1}{4} x^{2}+i b x, b \in \mathbb{R}_{*}$ & $M, I, D(1), G\left(e^{t}\right)-b e^{t} I, G\left(e^{-t}\right)+b e^{-t} I$ \\
$4 \mathrm{~b}$ & 1 & 2 & $-\frac{1}{4} x^{2}+i b x, b \in \mathbb{R}_{*}$ & $M, I, D(1), G(\cos t)+b(\sin t) I, G(\sin t)-b(\cos t) I$ \\
$4 \mathrm{c}$ & 1 & 2 & $i b x, b \in \mathbb{R}_{*}$ & $M, I, D(1), G(1)-b t I, G(t)-\frac{1}{2} b t^{2} I$ \\
5 & 3 & 0 & $c x^{-2}, c \in \mathbb{C}_{*}$ & $M, I, D(1), D(t), D\left(t^{2}\right)-\frac{1}{2} t I$ \\
6 & 3 & 2 & 0 & $M, I, D(1), D(t), D\left(t^{2}\right)-\frac{1}{2} t I, G(1), G(t)$ \\
\hline
\end{tabular}

Lie symmetry extensions given in Table 1 are maximal if the parameters involved satisfy the following conditions: In Case 1, the potential $V$ does not satisfy an equation of the form (9). In Case 2, the real-valued function $\gamma$ of $t$ is constrained by the condition $\gamma \neq c_{3}\left|c_{2} t^{2}+c_{1} t+c_{0}\right|^{-3 / 2}$ for any real constants $c_{0}, c_{1}, c_{2}$ and $c_{3}$ with $c_{0}, c_{1}$ and $c_{2}$ not vanishing simultaneously. In Case $3, V \neq b_{2} x^{2}+b_{1} x+b_{0}+c(x+a)^{-2}$ for any real constants $a, b_{2}$ and for any complex constants $b_{1}, b_{0}$ and $c$ with $c \operatorname{Im} b_{1}=0$. The real constant $b$ in Cases $4 \mathrm{a}-4 \mathrm{c}$ and the complex constant $c$ in Case 5 are nonzero. Further, $b>0 \bmod G^{\sim}$ in Cases $4 \mathrm{a}$ and $4 \mathrm{~b}$ and $b=1 \bmod G^{\sim}$ in Case $4 \mathrm{c}$.

Proof. We consider possible cases for the various values of $k_{1}$ and $k_{2}$.

$\boldsymbol{k}_{\mathbf{1}}=\boldsymbol{k}_{\mathbf{2}}=\mathbf{0}$. This is the general case with no extension, i.e., $\mathfrak{g}_{V}^{\text {ess }}=\mathfrak{g}^{\bigcap}$ (Case 1 of Table 1 ).

$\boldsymbol{k}_{\mathbf{1}}=\mathbf{0}, \boldsymbol{k}_{\mathbf{2}}=\mathbf{2}$. Lemmas 20 and 21 imply that up to $G^{\sim}$-equivalence the algebra $\mathfrak{g}_{V}^{\text {ess }}$ contains the vector fields $G(1)+\rho^{1} I$ and $G(t)+\rho^{2} I$, where $\rho^{1}$ is a smooth real-valued function of $t$ and $\rho^{2}=\int t \rho_{t}^{1} \mathrm{~d} t$. Integrating the classifying condition (9) for these vector fields with respect to $V$ gives $V=-i \rho_{t} x+\alpha(t)+i \beta(t)$, and $\alpha=\beta=0 \bmod G^{\sim}$. Denoting $-\rho_{t}$ by $\gamma$, we obtain $V=i \gamma(t) x, \rho^{1}=-\int \gamma \mathrm{d} t$ and $\rho^{2}=-\int t \gamma \mathrm{d} t$, which leads to Case 2 of Table 1.

Let us describe the values of $\gamma$ for which the Lie symmetry extension constructed is maximal. We substitute the potential $V=i \gamma(t) x$ into the classifying condition (9) and, after splitting with respect to $x$, derive the system

$$
\tau_{t t t}=0, \quad \chi_{t t}=0, \quad \sigma_{t}=0, \quad \rho_{t}=\chi \gamma-\frac{\tau_{t t}}{4}, \quad \tau \gamma_{t}+\frac{3}{2} \tau_{t} \gamma=0
$$


An additional Lie symmetry extension for such a potential may be realized only by vector fields with nonzero values of $\tau$. Then the integration of the first and last equations of the above system yields

$$
\tau=c_{2} t^{2}+c_{1} t+c_{0}, \quad \gamma=c_{3}|\tau|^{-3 / 2}=c_{3}\left|c_{2} t^{2}+c_{1} t+c_{0}\right|^{-3 / 2},
$$

where $c_{0}, c_{1}, c_{2}$ and $c_{3}$ are real constants. Therefore, Case 2 presents a maximal Lie symmetry extension if $\gamma \neq c_{3}\left|c_{2} t^{2}+c_{1} t+c_{0}\right|^{-3 / 2}$ for any real constants $c_{0}, c_{1}, c_{2}$ and $c_{3}$, where the constants $c_{0}, c_{1}$ and $c_{2}$ do not vanish simultaneously.

$\boldsymbol{k}_{1}=\mathbf{1}, \boldsymbol{k}_{2}=\mathbf{0}$. The algebra $\mathfrak{g}_{V}^{\text {ess }}$ necessarily contains a vector field $P^{0}$ of the form $P^{0}=D\left(\tau^{0}\right)+$ $G\left(\chi^{0}\right)+\sigma^{0} M+\rho^{0} I$, where all the parameter functions are real-valued functions of $t$ with $\tau^{0} \neq 0$. Pushing forward $P^{0}$ by a transformation from $\pi_{*} G^{\sim}$, we can set $\tau^{0}=1$ and $\chi^{0}=\sigma^{0}=\rho^{0}=0$. That is, up to $\pi_{*} G^{\sim}$-equivalence we can assume that $P^{0}=D(1)$, cf. Lemma 19, The classifying condition (9) for the vector field $P^{0}$ gives $V_{t}=0$, which implies Case 3 of Table 1 with an arbitrary time-independent potential $V$.

We now find the condition when the Lie symmetry extension obtained is really maximal. The presence of any additional extension means that the algebra $\mathfrak{g}_{V}^{\text {ess }}$ necessarily contains a vector field $Q=D(\tau)+G(\chi)+\sigma M+\rho I$ with $\tau_{t} \neq 0$ or $\chi \neq 0$. Substituting $Q$ into the classifying condition (9) and fixing a value of $t$ gives a linear ordinary differential equation with respect to $V=V(x)$. The general solution of any such equation is of the form $V=b_{2} x^{2}+b_{1} x+b_{0}+c(x+a)^{-2}$, where $a$ and $b_{2}$ are real constants and $b_{1}, b_{0}$ and $c$ are complex constants. Moreover, the constant $b_{1}$ is zero if $\tau_{t} \neq 0$ and, if $\tau_{t}=0$ and $\chi \neq 0$, we have $c=0$. Therefore, the Lie symmetry extension of Case 3 is maximal if and only if $V \neq b_{2} x^{2}+b_{1} x+b_{0}+c(x+a)^{-2}$ for any real constants $a$, $b_{2}$ and for any complex constant $b_{1}, b_{0}$ and $c$ with $c \operatorname{Im} b_{1}=0$.

$\boldsymbol{k}_{\mathbf{1}}=\mathbf{1}, \boldsymbol{k}_{\mathbf{2}}=\mathbf{2}$. In this case a basis of $\mathfrak{g}_{V}^{\text {ess }}$ consists of the vector fields $M, I, P^{0}=D\left(\tau^{0}\right)+$ $G\left(\chi^{0}\right)+\sigma^{0} M+\rho^{0} I$ and $Q^{p}=G\left(\chi^{p}\right)+\sigma^{p} M+\rho^{p} I$, where all the parameters are real-valued functions of $t$ with $\tau^{0} \neq 0$ and $\chi^{1}$ and $\chi^{2}$ being linearly independent. Here and in the following the indices $p$ and $q$ run from 1 to 2 and we sum over repeated indices. The vector field $P^{0}$ is reduced to $D(1)$ up to $\pi_{*} G^{\sim}$-equivalence, as in the previous case. The commutation relations of $\mathfrak{g}_{V}^{\text {ess }}$

$$
\begin{aligned}
& {\left[P^{0}, Q^{p}\right]=G\left(\chi_{t}^{p}\right)+\sigma_{t}^{p} M+\rho_{t}^{p} I=a_{p q} Q^{q}+a_{p 3} M+a_{p 4} I,} \\
& {\left[Q^{1}, Q^{2}\right]=\left(\chi^{1} \chi_{t}^{2}-\chi^{2} \chi_{t}^{1}\right) M=a_{0} M,}
\end{aligned}
$$

where $a_{p q}, a_{p 3}, a_{p 4}$ and $a_{0}$ are real constants, yield

$$
\chi_{t}^{p}=a_{p q} \chi^{q}, \quad \sigma_{t}^{p}=a_{p q} \sigma^{q}+a_{p 3}, \quad \rho_{t}^{p}=a_{p q} \rho^{q}+a_{p 4}, \quad \chi^{1} \chi_{t}^{2}-\chi^{2} \chi_{t}^{1}=a_{0} .
$$

The matrix $\left(a_{p q}\right)$ is not zero in view of the linear independence of $\chi^{1}$ and $\chi^{2}$. Moreover, the consistency of the system (10) implies that the trace of $\left(a_{p q}\right)$ is zero. Using equivalence transformations of time scaling, we can further scale the eigenvalues of the matrix $\left(a_{p q}\right)$ with the same nonzero real values. Replacing the vector fields $Q^{1}$ and $Q^{2}$ by their independent linear combinations leads to a matrix similarity transformation of $\left(a_{p q}\right)$. Hence, the matrix $\left(a_{p q}\right)$ can be assumed to be of one of the following real Jordan forms:

$$
\left(\begin{array}{cc}
1 & 0 \\
0 & -1
\end{array}\right), \quad\left(\begin{array}{cc}
0 & -1 \\
1 & 0
\end{array}\right), \quad\left(\begin{array}{ll}
0 & 0 \\
1 & 0
\end{array}\right)
$$

The further consideration of each of these forms consists of a few steps: We integrate the system of differential equations (10) for the chosen form of $\left(a_{p q}\right)$, which gives the components of the vector fields $Q^{1}$ and $Q^{2}$. From the classifying condition (9) for the basis vector fields of the algebra $\mathfrak{g}_{V}^{\text {ess }}$ we obtain three independent equations for the potential $V$, including the 
equation $V_{t}=0$. These equations must be solved jointly, and their consistency leads to additional constraints for constant parameters involved in $Q^{1}$ and $Q^{2}$. The expressions for both the vector fields $Q^{1}$ and $Q^{2}$ and the potential $V$ can be simplified by equivalence transformations and by changing the basis in the algebra $\mathfrak{g}_{V}^{\text {ess }}$ we can obtain expressions for $Q^{1}$ and $Q^{2}$ as in Cases $4 \mathrm{a}-4 \mathrm{c}$ of Table 1.

Integrating the system (10) for the first Jordan form, we obtain $\chi^{1}=b_{01} e^{t}, \sigma^{1}=b_{11} e^{t}-a_{13}$, $\rho^{1}=b_{12} e^{t}-a_{14}, \chi^{2}=b_{02} e^{-t}, \sigma^{2}=b_{21} e^{-t}+a_{23}, \rho^{2}=b_{22} e^{-t}+a_{24}$, where $a_{p 3}, a_{p 4}, b_{0 p}$ and $b_{p q}$ are real constants. Scaling the vector fields $Q^{1}$ and $Q^{2}$ and taking linear combinations of them with $M$ and $I$, we can set $b_{0 q}=1$ and $a_{p 3}=a_{p 4}=0$. The classifying condition (9) for the vector fields $P^{0}, Q^{1}$ and $Q^{2}$ leads to three independent equations in $V, V_{t}=0, V_{x}=\frac{1}{2} x-i b_{12}+b_{11}$ and $V_{x}=\frac{1}{2} x+i b_{22}-b_{21}$. These equations are consistent only if the constant parameters involved in $Q^{1}$ and $Q^{2}$ satisfy the constraints $-b_{12}=b_{22}=: b$ and $b_{11}=-b_{21}=:-\hat{b}$. Then the potential $V$ is of the form $V=\frac{1}{4}(x+2 \hat{b})^{2}+i b x+c_{1}+i c_{2}$ for some real constants $c_{1}$ and $c_{2}$. We apply the equivalence transformation (3) with $T=t, X^{0}=2 \hat{b}, \Sigma=-c_{1} t, \Upsilon=c_{2} t, \varepsilon=1$ and $\Phi=0$ and take a linear combination of the transformed vector field $P^{0}$ with $M$ and $I$. This allows us to set $\hat{b}=c_{1}=c_{2}=0$ and finally gives Case $4 \mathrm{a}$ of Table 1 .

In the same way, we consider the second Jordan form from (111). After integrating the corresponding system (10), we obtain $\chi^{1}=b_{01} \cos t-b_{02} \sin t, \sigma^{1}=b_{11} \cos t-b_{12} \sin t-a_{23}$, $\rho^{1}=b_{21} \cos t-b_{22} \sin t-a_{24}, \chi^{2}=b_{01} \sin t+b_{02} \cos t, \sigma^{2}=b_{11} \sin t+b_{12} \cos t+a_{13}$ and $\rho^{2}=b_{21} \sin t+b_{22} \cos t+a_{14}$, where $b_{0 q}$ and $b_{p q}$ are real constants. Combining the vector fields $Q^{1}$ and $Q^{2}$ with each other and with $M$ and $I$, we can put $b_{01}=1, b_{02}=0$ and $a_{p 3}=a_{p 4}=0$. Substituting the components of $P^{0}, Q^{1}$ and $Q^{2}$ that we obtain into the classifying condition (9) gives three independent equations in $V$,

$$
\begin{aligned}
& V_{t}=0, \\
& V_{x} \cos t=-\frac{1}{2} x \cos t+i\left(b_{21} \sin t+b_{22} \cos t\right)-b_{11} \sin t-b_{12} \cos t, \\
& V_{x} \sin t=-\frac{1}{2} x \sin t-i\left(b_{21} \cos t-b_{22} \sin t\right)+b_{11} \cos t-b_{12} \sin t
\end{aligned}
$$

with the consistency condition $b_{11}=b_{21}=0$. We denote $b_{12}=: \hat{b}$ and $b_{22}=: b$. Any solution of the above equations for $V$ can be written as $V=-\frac{1}{4}(x+2 \hat{b})^{2}+i b x+c_{1}+i c_{2}$ for some real constants $c_{1}$ and $c_{2}$. By applying the equivalence transformation (3) with $T=t, X^{0}=2 \hat{b}$, $\Sigma=-c_{1} t, \Upsilon=c_{2} t, \varepsilon=1$ and $\Phi=0$ and taking a linear combination of the transformed vector field $P^{0}$ with $M$ and $I$, we can put $\hat{b}=c_{1}=c_{2}=0$. This yields Case $4 \mathrm{~b}$ of Table 1 .

Finally, the general solution of the system (10) for the last Jordan form of the matrix $\left(a_{p q}\right)$ is $\chi^{1}=b_{01}, \sigma^{1}=a_{13} t+b_{11}, \rho^{1}=a_{14} t+b_{21}, \chi^{2}=b_{01} t+b_{02}, \sigma^{2}=\frac{1}{2} a_{13} t^{2}+\left(a_{23}+b_{11}\right) t+b_{12}$, $\rho^{2}=\frac{1}{2} a_{14} t^{2}+\left(a_{24}+b_{21}\right) t+b_{22}$, where $b_{0 q}$ and $b_{p q}$ are real constants. The constants $b_{p q}$ and $b_{02}$ can be put equal to zero and $b_{01}=1$ by taking a linear combination of the vector fields $Q^{1}$ and $Q^{2}$ with each other and with $M$ and $I$. Then we successively evaluate the classifying condition (9) for the components of the vector fields $P^{0}, Q^{1}$ and $Q^{2}$. This gives the following equations for $V$ :

$$
V_{t}=0, \quad t V_{x}=-i a_{14} t+a_{13} t, \quad t V_{x}=-i a_{14} t+a_{13} t-i a_{24}+a_{23},
$$

which are consistent if and only if $a_{23}=a_{24}=0$. Any solution of these equations is of the form $V=i b x+\hat{b} x+c_{1}+i c_{2}$, where $c_{1}$ and $c_{2}$ are real constants and we denote $a_{13}=: \hat{b}$ and $a_{14}=:-b$. Next we apply the equivalence transformation (3) with $T=t, X^{0}=-\hat{b} t^{2}, \Sigma=\frac{1}{3} \hat{b}^{2} t^{3}-c_{1} t$, $\Upsilon=\frac{1}{3} b \hat{b} t^{3}+c_{2} t, \varepsilon=1$ and $\Phi=0$ and take a linear combination of the vector fields $P^{0}$ with $Q^{2}$, $M$ and $I$. In this way the constants $\hat{b}, c_{1}$ and $c_{2}$ are set equal to zero, which gives Case $4 \mathrm{c}$ of Table 1. 
The Lie symmetry extensions presented in Cases $4 \mathrm{a}-4 \mathrm{c}$ of Table 1 are really maximal if $b \neq 0$. Moreover, up to $G^{\sim}$-equivalence we can set $b=1$ in Case $2 \mathrm{a}$ and $b>0$ in Cases $2 \mathrm{~b}$ and 2c; cf. the proof of Theorem 32 .

$\boldsymbol{k}_{\mathbf{1}} \geqslant \mathbf{2}, \boldsymbol{k}_{\mathbf{2}}=\mathbf{0}$. Lemma 19 implies that up to $\pi_{*} G^{\sim}$-equivalence the algebra $\mathfrak{g}_{V}^{\text {ess }}$ contains at least two operators $P^{0}=D(1)$ and $P^{1}=D(t)+G\left(\chi^{1}\right)+\sigma^{1} M+\rho^{1} I$. Here we also annihilate the tail of $P^{0}$ with pushing forward $P^{0}$ by a transformation from $\pi_{*} G^{\sim}$. As $\left[P^{0}, P^{1}\right] \in \mathfrak{g}_{V}^{\text {ess }}$, we have

$$
\left[P^{0}, P^{1}\right]=D(1)+G\left(\chi_{t}^{1}\right)+\sigma_{t}^{1} M+\rho_{t}^{1} I=P^{0}+a_{1} M+b_{1} I
$$

for some constants $a_{1}$ and $b_{1}$. Collecting components in the above equality gives the system $\chi_{t}^{1}=0, \sigma_{t}^{1}=a_{1}, \rho_{t}^{1}=b_{1}$ with the general solution $\chi^{1}=a_{2}, \sigma^{1}=a_{1} t+a_{0}$ and $\rho^{1}=b_{1} t+b_{0}$, where $a_{2}, a_{0}$ and $b_{0}$ are real constants of integration. Pushing forward $P^{0}$ and $P^{1}$ with $\mathcal{G}_{*}\left(2 a_{2}\right)$, $\mathcal{M}_{*}\left(-a_{1} t\right)$ and $\mathcal{I}_{*}\left(-b_{1} t\right)$ and taking a linear combination of $P^{0}$ and $P^{1}$ with $M$ and $I$, we find that we can set the constants $a_{0}, a_{1}, a_{2}, b_{0}$ and $b_{1}$ to zero. Therefore, the basis vector field $P^{1}$ reduces to the form $P^{1}=D(t)$, whereas the forms of $P^{0}, M$ and $I$ are preserved.

The classifying condition (9) for $P^{0}=D(1)$ and $P^{1}=D(t)$ gives two independent equations in $V, V_{t}=0$ and $x V_{x}+2 V=0$. Integrating these equations gives $V=c x^{-2}$, where $c$ is a complex constant. If $c=0$, then $k_{2}>0$, which contradicts the case assumption $k_{2}=0$. Thus, the constant $c$ is nonzero. We find the maximal Lie invariance algebra in this case. We substitute $V=c x^{-2}$ with $c \neq 0$ into the classifying condition (9) and derive the system of differential equations for functions parameterizing vector fields from $\mathfrak{g}_{V}^{\text {ess }}, \tau_{t t t}=0, \chi=0, \sigma_{t}=0$, $\rho_{t}=-\frac{1}{4} \tau_{t t}$. The solution of the above system implies that the algebra $\mathfrak{g}_{V}^{\text {ess }}$ is spanned by $M, I$, $P^{0}, P^{1}$ and one more vector field $P^{2}=D\left(t^{2}\right)-\frac{1}{2} t I$, which gives Case 5 of Table 1 .

$\boldsymbol{k}_{\mathbf{1}} \geqslant \mathbf{2}, \boldsymbol{k}_{\mathbf{2}}=\mathbf{2}$. In this case, the algebra $\mathfrak{g}_{V}^{\text {ess }}$ necessarily contains the vector fields $M, I, P^{l}=$ $D\left(\bar{\tau}^{l}\right)+G\left(\bar{\chi}^{l}\right)+\bar{\sigma}^{l} M+\bar{\rho}^{l}, l=0,1$ and $Q^{p}=G\left(\chi^{p}\right)+\sigma^{p} M+\rho^{p} I$, where all the parameters are real-valued smooth functions of $t$ with $\bar{\tau}^{0}$ and $\bar{\tau}^{1}$ (resp. $\chi^{1}$ and $\chi^{2}$ ) being linearly independent. Recall that the indices $p$ and $q$ run from 1 to 2 , and we sum over repeated indices. As in the previous case, up to $\pi_{*} G^{\sim}$-equivalence the vector fields $P^{0}$ and $P^{1}$ reduce to the form $P^{0}=D(1)$ and $P^{1}=D(t)+G\left(\bar{\chi}^{1}\right)+\bar{\sigma}^{1} M+\bar{\rho}^{1} I$.

Since the algebra $\mathfrak{g}_{V}^{\text {ess }}$ is closed with respect to the Lie bracket of vector fields, we have $\left[P^{l}, Q^{p}\right] \in \mathfrak{g}_{V}^{\text {ess }}$, i.e.,

$$
\begin{aligned}
& {\left[P^{0}, Q^{p}\right]=G\left(\chi_{t}^{p}\right)+\sigma_{t}^{p} M+\rho_{t}^{p} I=a_{p q} Q^{q}+a_{p 3} M+a_{p 4} I} \\
& {\left[P^{1}, Q^{p}\right]=d_{p q} Q^{q}+d_{p 3} M+d_{p 4} I,}
\end{aligned}
$$

where $a_{p q}, a_{p 3}, a_{p 4}, d_{p q}, d_{p 3}$ and $d_{p 4}$ are real constants. Using the above commutation relations with $P^{0}$ in the same way as in the case $k_{1}=1, k_{2}=2$, we derive three inequivalent cases for the vector fields $Q^{p}$ depending on the Jordan forms of the matrix $\left(a_{p q}\right)$ presented in (11). For the first and second Jordan forms, the commutators $\left[P^{1}, Q^{p}\right]$ do not belong to the linear span of $P^{0}, P^{1}, Q^{1}, Q^{2}, M$ and $I$. Hence these cases are irrelevant.

For the last Jordan form from (11), up to $G^{\sim}$-equivalence and up to linear combining of the above vector fields, we can further assume that $Q^{1}=G(1)-b t I, Q^{2}=G(t)-\frac{1}{2} b t^{2} I$ and $V=i b x$ for some real constant $b$. We expand the commutation relation for the vector fields $P^{1}$ and $Q^{1}$ :

$$
\left[P^{1}, Q^{1}\right]=-\frac{1}{2} G(1)-b t e^{t} I-\bar{\chi}_{t}^{1} M=d_{11} Q^{1}+d_{12} Q^{2}+d_{13} M+d_{14} I,
$$

and equating components gives $b=0$, i.e., $V=0$. Substituting the value $V=0$ into the classifying condition (91) and splitting with respect to $x$ yields the system of differential equations $\tau_{t t t}=0, \chi_{t t}=0, \sigma_{t}=0, \rho_{t}=-\frac{1}{4} \tau_{t t}$. The solution of this system for $V=0$ shows that the algebra $\mathfrak{g}_{V}^{\text {ess }}$ is spanned by the vector fields presented in Case 6 of Table 1 . 
Remark 25. It might be convenient to completely describe properties of appropriate subalgebras before their classification but often such an approach is not justified. Thus, Lemma 19 shows that the invariant $k_{1}$ is not greater than three, i.e., $k_{1} \in\{0,1,2,3\}$. As we proved in Theorem 24, this invariant cannot be equal to two. The reason is that any (finite-dimensional) subalgebra $\mathfrak{s}$ of $\mathfrak{g}_{\langle>}^{\text {ess }}$ with $\operatorname{dim} \pi_{*}^{0} \mathfrak{s}=2$ is not appropriate since the condition of extension maximality is not satisfied. Therefore, Lemma 19 could be strengthened by the constraint $k_{1} \in\{0,1,3\}$. At the same time, the proof of the condition $k_{1} \neq 2$ needs realizing the major part of the group classification of the class (1).

\section{Alternative proof}

Here we present an alternative way of classifying Lie symmetry extensions in the class (11), in which the invariant $k_{2}$ is considered as leading. The case $k_{2}=0$, after partitioning into the subcases $k_{1}=0, k_{1}=1$ or $k_{1} \geqslant 2$, results in the same extensions as presented in Table 1 for these values of $k_{1}$ and $k_{2}$.

Let us consider the case $k_{2}=2$ more closely. Lemmas 20 and 21 imply that, up to $G^{\sim}$ equivalence, the algebra $\mathfrak{g}_{V}^{\text {ess }}$ contains the vector fields $G(1)+\rho^{1} I$ and $G(t)+\rho^{2} I$, where $\rho^{1}$ is a smooth real-valued function of $t$ and $\rho^{2}=\int t \rho_{t}^{1} \mathrm{~d} t$. Integrating the classifying condition (9) for these vector fields with respect to $V$ gives $V=-i \rho_{t} x+\alpha(t)+i \beta(t)$, and $\alpha=\beta=0 \bmod G^{\sim}$. Denoting $-\rho_{t}$ by $\gamma$, we obtain $V=i \gamma(t) x$. Thus, we carry out the group classification of the subclass of equations from the class (1) with potentials of this form, i.e.,

$$
i \psi_{t}+\psi_{x x}+i \gamma(t) x \psi=0,
$$

where $\gamma(t)$ is an arbitrary real-valued function of $t$, which will be taken as the arbitrary element of the subclass instead of $V$. For potentials of the above form, the equation (3c) splits with respect to $x$ and gives the system of differential equations

$$
2 T_{t t t} T_{t}-3 T_{t t}^{2}=0, \quad\left(\frac{X_{t}^{0}}{T_{t}}\right)_{t}=0, \quad \Sigma_{t}=\frac{\left(X_{t}^{0}\right)^{2}}{4 T_{t}}, \quad \Upsilon_{t}=-\frac{T_{t t}}{4 T_{t}}-\varepsilon \frac{\gamma X^{0}}{\left|T_{t}\right|^{1 / 2}},
$$

whose general solution is

$$
T=\frac{a_{1} t+a_{0}}{a_{3} t+a_{2}}, \quad X^{0}=b_{1} T+b_{0}, \quad \Sigma=\frac{b_{1}^{2}}{4} T+c_{1}, \quad \Upsilon=-\frac{1}{4} \ln \left|T_{t}\right|-\varepsilon \int \frac{\gamma X^{0}}{\left|T_{t}\right|^{1 / 2}} \mathrm{~d} t+c_{0},
$$

where $a_{i}, i=0, \ldots, 3, b_{j}$ and $c_{j}, j=0,1$, are real constants with $a_{1} a_{2}-a_{0} a_{3} \neq 0$ and the integral denotes a fixed primitive function for the integrand. Since the constants $a_{i}, i=0, \ldots, 3$, are defined up to a nonzero constant multiplier (and thus only three of the constants are essential), we set $a_{1} a_{2}-a_{0} a_{3}=\operatorname{sgn} T_{t}:=\varepsilon^{\prime}= \pm 1$.

To single out the equivalence groupoid $\mathcal{G}_{(12)}^{\sim}$ of the subclass (12) from the equivalence groupoid $\mathcal{G}^{\sim}$ of the whole class (11), we substitute the above values of $T, X^{0}, \Sigma$ and $\Upsilon$ into (3) and obtain the following statement:

Theorem 26. The equivalence groupoid $\mathcal{G}_{(12)}^{\sim}$ of the subclass (12) consists of triples of the form $(\gamma, \tilde{\gamma}, \varphi)$, where $\varphi$ is a point transformation in the space of variables, whose components are

$$
\begin{aligned}
& \tilde{t}=T:=\frac{a_{1} t+a_{0}}{a_{3} t+a_{2}}, \quad \tilde{x}=\varepsilon\left|T_{t}\right|^{1 / 2} x+b_{1} T+b_{0} \\
& \tilde{\psi}=\frac{c}{\left|T_{t}\right|^{1 / 4}} \exp \left(\frac{i}{8} \frac{T_{t t}}{\left|T_{t}\right|} x^{2}+\frac{i}{2} \varepsilon b_{1}\left|T_{t}\right|^{1 / 2} x-\varepsilon \int \gamma \frac{b_{1} T+b_{0}}{\left|T_{t}\right|^{1 / 2}} \mathrm{~d} t+i \frac{b_{1}^{2}}{4} T\right)(\hat{\psi}+\hat{\Phi}),
\end{aligned}
$$


the transformed parameter $\tilde{\gamma}$ is given in terms of $\gamma$ as

$$
\tilde{\gamma}=\frac{\varepsilon \varepsilon^{\prime}}{\left|T_{t}\right|^{3 / 2}} \gamma
$$

$a_{0}, a_{1}, a_{2}, a_{3}, b_{0}$ and $b_{1}$ are arbitrary real constants with $a_{1} a_{2}-a_{0} a_{3}=: \varepsilon^{\prime}= \pm 1, c$ is a nonzero complex constant, $\Phi=\Phi(t, x)$ is an arbitrary solution of the initial equation, $\varepsilon= \pm 1$.

Corollary 27. The (usual) equivalence group $G_{(12)}^{\sim}$ of the subclass (12) consists of point transformations of the form (13) with $b_{0}=b_{1}=0$ and $\Phi=0$.

Proof. We argue in a similar way to Corollary 8 , since each transformation from $G_{(12)}^{\sim}$ generates a family of admissible transformations in the subclass (12), it is necessarily of the form (13). There is only one common solution for the equations from the subclass (12): the zero function. Hence the independence of the transformation components for the variables on the arbitrary element $\gamma$ is equivalent to the conditions $b_{0}=b_{1}=0$ and $\Phi=0$.

Corollary 28. The equivalence algebra of the subclass (12) is the algebra

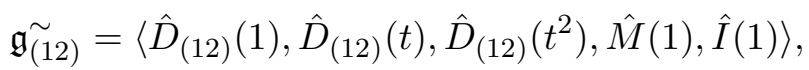

where, as in Corollary 11, $\hat{M}(1)=i\left(\psi \partial_{\psi}-\psi^{*} \partial_{\psi^{*}}\right), \hat{I}(1)=\psi \partial_{\psi}+\psi^{*} \partial_{\psi^{*}}$, and

$$
\hat{D}_{\text {(12) }}(\tau)=\tau \partial_{t}+\frac{1}{2} \tau_{t} x \partial_{x}+\frac{1}{8} \tau_{t t} x^{2} \hat{M}(1)-\frac{1}{4} \tau_{t} \hat{I}(1)-\frac{3}{2} \tau_{t} \gamma \partial_{\gamma} .
$$

The proof is analogous to that of Corollary 11,

Corollary 29. For each $\gamma=\gamma(t)$, the equation $\mathcal{L}_{V}$ with $V=i \gamma x$ admits the group $G_{V}^{\text {unf }}$ of point symmetry transformations of the form (13a) -113b) with $T=t$ and $\varepsilon=1$.

Proof. The relation (13c) obviously implies that for each fixed value of the arbitrary element $\gamma$, transformations of the form (13a) $-(13 \mathrm{~b})$ with $T=t$ and $\varepsilon=1$ leave this value invariant. Other transformations are point symmetries of $\mathcal{L}_{V}$ with $V=i \gamma x$ only for some values of $\gamma$.

Corollary 30. The subclass (12) is uniformly semi-normalized with respect to the family of uniform point symmetry groups $\left\{G_{V}^{\mathrm{unf}}\right\}$ of equations from this subclass and the subgroup $H$ of $G_{\tilde{(12)}}^{\sim}$ singled out by the constraint $c=1$.

Proof. It is obvious that for any $V$ the intersection of $\pi_{*} H$ and $G_{V}^{\text {unf }}$ consists of the identity transformation only. Consider an arbitrary admissible transformation $(\gamma, \tilde{\gamma}, \varphi)$ in the subclass (12), which maps the equation $\mathcal{L}_{V}$ with $V=i \gamma(t) x$ to the equation $\mathcal{L}_{\tilde{V}}$ with $\tilde{V}=i \tilde{\gamma}(\tilde{t}) \tilde{x}$. Then $\varphi$ is of the form (13a) - (13b) and thus $G_{\tilde{V}}^{\mathrm{unf}}=\varphi G_{V}^{\mathrm{unf}} \varphi^{-1}$. We denote the dependence of $\varphi$ on the transformation parameters appearing in (13a) (13b) by writing $\varphi=\varphi\left(T, \varepsilon, b_{1}, b_{0}, c, \Phi\right)$. It is obvious that $\varphi=\varphi^{2} \varphi^{0} \varphi^{1}$, where $\varphi^{1}=\varphi(t, 1,0,0,1, \Phi) \in G_{V}^{\mathrm{unf}}, \varphi^{2}=\varphi\left(t, 1, b_{1}, b_{0}, c, 0\right) \in G_{\tilde{V}}^{\mathrm{unf}}$, and the transformation $\varphi^{0}=\varphi(T, \varepsilon, 0,0,1,0)$, prolonged to $\gamma$ according to (13c), belongs to $H$.

Applying Theorem 14 to equations from the subclass (12), we consider the classifying condition (9) for the associated form of potentials, $V=i \gamma(t) x$, and split this condition with respect to $x$. As a result, we obtain the following system of differential equations for the parameters of Lie symmetry vector fields:

$$
\tau_{t t t}=0, \quad \chi_{t t}=0, \quad \sigma_{t}=0, \quad \rho_{t}=-\gamma \chi-\frac{1}{4} \tau_{t t},
$$

as well as the classifying condition

$$
\left(\gamma|\tau|^{3 / 2}\right)_{t}=0 .
$$

It is then clear that the kernel invariance algebra $\mathfrak{g}_{(12)}^{\cap}$ of the subclass (12) is spanned by the vector fields $M$ and $I$. 
Theorem 31. The maximal Lie invariance algebra $\mathfrak{g}_{V}$ of an equation $\mathcal{L}_{V}$ for $V=i \gamma(t) x$ is spanned by the vector fields $D_{\underline{\underline{12}}(\tau)}(\tau), G(1)+\rho^{1} I, G(t)+\rho^{2} I, M, I, Z\left(\eta^{0}\right)$, where

$$
D_{\overline{112}}(\tau):=D(\tau)-\frac{1}{4} \tau_{t} I=\tau \partial_{t}+\frac{1}{2} \tau_{t} x \partial_{x}+\frac{1}{8} \tau_{t t} x^{2} M-\frac{1}{4} \tau_{t} I
$$

the parameter $\tau$ runs through the set $\mathfrak{P}_{\gamma}$ of quadratic polynomials in $t$ that satisfy the classifying condition (15), $\rho^{1}=-\int \gamma(t) \mathrm{d} t, \rho^{2}=-\int t \gamma(t) \mathrm{d} t$ and $\eta^{0}$ runs through the solution set of the equation $\mathcal{L}_{V}$.

Each equation $\mathcal{L}_{V}$ with $V=i \gamma(t) x$, belonging to the subclass (12), is invariant with respect to the Lie algebra $\mathfrak{g}_{V}^{\text {unf }}=\left\langle G(1)+\rho^{1} I, G(t)+\rho^{2} I, M, I, Z\left(\eta^{0}\right)\right\rangle$ of the group $G_{V}^{\text {unf }}$, where $\eta^{0}$ again runs through the solution set of the equation $\mathcal{L}_{V}$. Such algebras have a similar structure for all equations from the subclass. The commutation relations between vector fields from $\mathfrak{g}_{\langle\rangle}$imply that the essential part $\mathfrak{g}_{V}^{\text {ess }}$ of $\mathfrak{g}_{V}$ admits the representation $\mathfrak{g}_{V}^{\text {ess }}=\mathfrak{g}_{V}^{\text {ext }} \notin\left(\mathfrak{g}_{V}^{\text {unf }} \cap \mathfrak{g}_{\langle\rangle}^{\text {ess }}\right)$, where $\mathfrak{g}_{V}^{\text {ext }}=\left\{D_{(12)}(\tau) \mid \tau \in \mathfrak{P}_{\gamma}\right\}$ is a subalgebra of $\mathfrak{g}_{V}^{\text {ess }}$, and $\mathfrak{g}_{V}^{\text {unf }}$ is an ideal of $\mathfrak{g}_{V}^{\text {ess }} \cap \mathfrak{g}_{\langle\rangle}^{\text {ess }}$. Interpreting the above representation, we can say that the algebra $\mathfrak{g}_{V}^{\text {ess }}$ is obtained by extending the algebra $\mathfrak{g}_{V}^{\text {unf }} \cap \mathfrak{g}_{\langle\rangle}^{\text {ess }}$ with elements of $\mathfrak{g}_{V}^{\text {ext }}$.

Consider the linear span

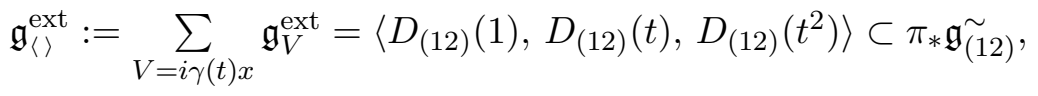

where $\pi$ is the projection of the joint space of the variables and the arbitrary element on the space of the variables only. The algebra $\mathfrak{g}_{\langle\rangle}^{\text {ext }}$ is isomorphic to the algebra $\operatorname{sl}(2, \mathbb{R})$. The pushforwards of vector fields from $\mathfrak{g}_{\langle\rangle}^{\text {ext }}$ by transformations from the group $\pi_{*} G_{\tilde{(12)}}^{\sim}$ constitute the inner automorphism group $\operatorname{Inn}\left(\mathfrak{g}_{\langle\rangle}^{\text {ext }}\right)$ of the algebra $\mathfrak{g}_{\langle\rangle}^{\text {ext }}$. The action of $G_{(12)}^{\sim}$ on equations from the subclass (12) induces the action of $\operatorname{Inn}\left(\mathfrak{g}_{\langle\rangle}^{\text {ext }}\right)$ on the subalgebras of the algebra $\mathfrak{g}_{\langle\rangle}^{\text {ext }}$. Consequently, the classification of possible Lie symmetry extensions in the subclass (12) reduces to the classification of subalgebras of the algebra $\operatorname{sl}(2, \mathbb{R})$, which is well known.

Theorem 32. A complete list of $G_{(\overline{12})}^{\sim}$-inequivalent (and, therefore, $\mathcal{G}_{(12)}^{\sim}$-inequivalent) Lie symmetry extensions in the subclass (12) is given by Table 2.

Table 2. Results of the group classification of the subclass (12).

\begin{tabular}{|c|c|c|l|}
\hline no. & $k_{1}$ & $V$ & \multicolumn{1}{c|}{ Basis of $\mathfrak{g}_{V}^{\text {ess }}$} \\
\hline 1 & 0 & $i \gamma(t) x$ & $M, I, G(1)-\left(\int \gamma(t) \mathrm{d} t\right) I, G(t)-\left(\int t \gamma(t) \mathrm{d} t\right) I$ \\
$2 \mathrm{a}$ & 1 & $i b x, b \in \mathbb{R}_{*}$ & $M, I, G(1)-b t I, G(t)-\frac{1}{2} b t^{2} I, D(1)$ \\
$2 \mathrm{~b}$ & 1 & $i b|t|^{-3 / 2} x, b \in \mathbb{R}_{*}$ & $M, I, G(1)+2 b t|t|^{-3 / 2} I, G(t)-2 b|t|^{1 / 2} I, D(t)$ \\
$2 \mathrm{c}$ & 1 & $i b\left(t^{2}+1\right)^{-3 / 2} x, b \in \mathbb{R}_{*}$ & $M, I, G(1)-b t\left(t^{2}+1\right)^{-1 / 2} I, G(t)+b\left(t^{2}+1\right)^{-1 / 2} I$, \\
& & & $D\left(t^{2}+1\right)-\frac{1}{2} t I$ \\
3 & 3 & 0 & $M, I, G(1), G(t), D(1), D(t), D\left(t^{2}\right)-\frac{1}{2} t I$ \\
\hline
\end{tabular}

Lie symmetry extension given in Case 1 of Table 2 is maximal if and only if the arbitrary element $\gamma$ is of the form $\gamma \neq c_{3}\left|c_{2} t^{2}+c_{1} t+c_{0}\right|^{-3 / 2}$ for any real constants $c_{0}, c_{1}, c_{2}$ and $c_{3}$ with $c_{0}, c_{1}$ and $c_{2}$ not vanishing simultaneously. $b=1 \bmod G^{\sim}$ in Case $2 \mathrm{a}$ and $b>0 \bmod G^{\sim}$ in Cases $2 \mathrm{~b}$ and $2 \mathrm{c}$. 
Proof. An optimal set of subalgebras of the algebra $\mathfrak{g}_{\langle\rangle}^{\text {ext }}$ is given by

$\{0\},\langle D(1)\rangle,\langle D(t)\rangle,\left\langle D\left(t^{2}+1\right)-\frac{1}{2} t I\right\rangle,\langle D(1), D(t)\rangle,\left\langle D(1), D(t), D\left(t^{2}+1\right)-\frac{1}{2} t I\right\rangle$.

The zero subalgebra gives the general case with no extension of $\mathfrak{g}_{V}^{\text {unf }}$, which is Case 1 of Table 2.

For the one-dimensional subalgebras, we substitute the corresponding values of $\tau, \tau=1$, $\tau=t$ and $\tau=t^{2}+1$ into the classifying condition (15), integrate the resulting equations with respect to $\gamma$ and obtain Cases 2a-2c of Table 2, respectively. Using equivalence transformations that do not change the form of $\gamma$, we can set $b=1$ in Case $2 \mathrm{a}$ and $b>0$ in Cases $2 \mathrm{~b}$ and 2c.

Similarly, the classifying condition (15) for the two-dimensional subalgebra gives an overdetermined system of two equations with $\tau=1$ and $\tau=t$, for which the only solution is $\gamma=0$. The maximal extension of $\mathfrak{g}_{V}^{\text {unf }}$ for $\gamma=0$ is three-dimensional and is given by the last subalgebra of the list. This gives Case 3 of Table 1 .

All cases presented in Table 2 are related to those of Table 1. In the symbol T.N, used in the following, $\mathrm{T}$ denotes the table number and $\mathrm{N}$ is the case number (in Table $\mathrm{T}$ ). Thus, Cases 2.1, 2.2a and 2.3 coincide with Cases 1.2, 1.4c and 1.6, respectively. Some cases are connected via equivalence transformations, which are of the form (3),

$$
\begin{array}{ll}
2.2 b \rightarrow 1.4 a: & T=\frac{\operatorname{sgn} t}{4} \ln |t|, \quad X^{0}=\Sigma=\Upsilon=0, \quad \Phi=0 ; \\
2.2 c \rightarrow 1.4 b: & T=\arctan t, \quad X^{0}=\Sigma=\Upsilon=0, \quad \Phi=0 .
\end{array}
$$

Thus, the result of group classification of the class (11) can be reformulated with involving Table 2 .

Corollary 33. A complete list of inequivalent Lie symmetry extensions in the class (1) is exhausted by Cases 1, 3 and 5 of Table 1 and the cases collected in Table 2.

\section{Subclass with real-valued potentials}

We derive results on group analysis of the subclass $\mathrm{Sch}_{\mathbb{R}}$ of equations of the form (1) with realvalued potentials using those for the whole class (1). The condition that potentials are real valued leads to additional constraints for transformations and infinitesimal generators.

Theorem 34. The equivalence groupoid $\mathcal{G}_{\mathbb{R}}^{\sim}$ of the subclass $\mathrm{Sch}_{\mathbb{R}}$ consists of triples of the form $(V, \tilde{V}, \varphi)$, where $\varphi$ is a point transformation in the space of variables, whose components are

$$
\begin{aligned}
& \tilde{t}=T, \quad \tilde{x}=\varepsilon\left|T_{t}\right|^{1 / 2} x+X^{0}, \\
& \tilde{\psi}=\frac{a}{\left|T_{t}\right|^{1 / 4}} \exp \left(\frac{i}{8} \frac{T_{t t}}{\left|T_{t}\right|} x^{2}+\frac{i}{2} \frac{\varepsilon \varepsilon^{\prime} X_{t}^{0}}{\left|T_{t}\right|^{1 / 2}} x+i \Sigma\right)(\hat{\psi}+\hat{\Phi}),
\end{aligned}
$$

the transformed potential $\tilde{V}$ is expressed in terms of $V$ as

$$
\tilde{V}=\frac{V}{\left|T_{t}\right|}+\frac{2 T_{t t t} T_{t}-3 T_{t t}^{2}}{16 \varepsilon^{\prime} T_{t}^{3}} x^{2}+\frac{\varepsilon \varepsilon^{\prime}}{2\left|T_{t}\right|^{1 / 2}}\left(\frac{X_{t}^{0}}{T_{t}}\right)_{t} x-\frac{\left(X_{t}^{0}\right)^{2}}{4 T_{t}^{2}}+\frac{\Sigma_{t}}{T_{t}},
$$

$T=T(t), X^{0}=X^{0}(t)$ and $\Sigma=\Sigma(t)$ are arbitrary smooth real-valued functions of $t$ with $T_{t} \neq 0$ and $\Phi=\Phi(t, x)$ is an arbitrary solution of the initial equation. a is a nonzero real constant, $\varepsilon= \pm 1$ and $\varepsilon^{\prime}=\operatorname{sgn} T_{t}$.

Corollary 35. The subclass $\mathrm{Sch}_{\mathbb{R}}$ is uniformly semi-normalized with respect to linear superposition of solutions. Its equivalence group $G_{\mathbb{R}}^{\sim}$ consists of point transformations of the form (16) with $\Phi=0$. 
Corollary 36. The equivalence algebra of the subclass $\mathrm{Sch}_{\mathbb{R}}$ is the algebra

$$
\mathfrak{g}_{\mathbb{R}}^{\sim}=\left\langle\hat{D}_{\mathbb{R}}(\tau), \hat{G}_{\mathbb{R}}(\chi), \hat{M}_{\mathbb{R}}(\sigma), \hat{I}_{\mathbb{R}}\right\rangle
$$

where $\tau, \chi$ and $\sigma$ run through the set of smooth real-valued functions of $t$. The vector fields $\hat{D}_{\mathbb{R}}(\tau), \hat{G}_{\mathbb{R}}(\chi), \hat{M}_{\mathbb{R}}(\sigma)$ and $\hat{I}_{\mathbb{R}}$ are given by

$$
\begin{aligned}
& \hat{D}_{\mathbb{R}}(\tau)=\tau \partial_{t}+\frac{1}{2} \tau_{t} x \partial_{x}+\frac{i}{8} \tau_{t t} x^{2}\left(\psi \partial_{\psi}-\psi^{*} \partial_{\psi^{*}}\right)-\frac{1}{4} \tau_{t} \hat{I}_{\mathbb{R}}-\left(\tau_{t} V-\frac{1}{8} \tau_{t t t} x^{2}\right) \partial_{V}, \\
& \hat{G}_{\mathbb{R}}(\chi)=\chi \partial_{x}+\frac{i}{2} \chi_{t} x\left(\psi \partial_{\psi}-\psi^{*} \partial_{\psi^{*}}\right)+\frac{\chi_{t t}}{2} x \partial_{V}, \\
& \hat{M}_{\mathbb{R}}(\sigma)=i \sigma\left(\psi \partial_{\psi}-\psi^{*} \partial_{\psi^{*}}\right)+\sigma_{t} \partial_{V}, \quad \hat{I}_{\mathbb{R}}=\psi \partial_{\psi}+\psi^{*} \partial_{\psi^{*}} .
\end{aligned}
$$

Corollary 37. A (1+1)-dimensional linear Schrödinger equation of the form (11) with a realvalued potential $V$ is equivalent to the free linear Schrödinger equation with respect to a point transformation if and only if the potential is a quadratic polynomial in $x$, i.e., $V=\gamma^{2}(t) x^{2}+$ $\gamma^{1}(t) x+\gamma^{0}(t)$ for some smooth real-valued functions $\gamma^{0}, \gamma^{1}$ and $\gamma^{2}$ of $t$.

A study of the determining equations for Lie symmetries of equations from the subclass $\mathrm{Sch}_{\mathbb{R}}$ shows that the classifying condition in this case is of the form (9) with $\rho_{t}=-\frac{1}{4} \tau_{t t}$,

$$
\tau V_{t}+\left(\frac{1}{2} \tau_{t} x+\chi\right) V_{x}+\tau_{t} V=\frac{1}{8} \tau_{t t t} x^{2}+\frac{1}{2} \chi_{t t} x+\sigma_{t}
$$

The kernel invariance algebra $\mathfrak{g}_{\mathbb{R}}^{\bigcap}$ of the subclass $\mathrm{Sch}_{\mathbb{R}}$ coincides with the kernel invariance algebra $\mathfrak{g}^{\cap}$ of the whole class (1), cf. Proposition 1.

Theorem 38. The maximal Lie invariance algebra $\mathfrak{g}_{V}$ of an equation $\mathcal{L}_{V}$ from the subclass $\mathrm{Sch}_{\mathbb{R}}$ is spanned by the vector fields $D_{\mathbb{R}}(\tau), G(\chi), I, \sigma M$ and $Z\left(\eta^{0}\right)$, where

$$
\begin{aligned}
& D_{\mathbb{R}}(\tau):=D(\tau)-\frac{1}{4} \tau_{t} I=\tau \partial_{t}+\frac{1}{2} \tau_{t} x \partial_{x}+\frac{1}{8} \tau_{t t} x^{2} M-\frac{1}{4} \tau_{t} I, \quad G(\chi)=\chi \partial_{x}+\frac{1}{2} \chi_{t} x M, \\
& M=i \psi \partial_{\psi}-i \psi^{*} \partial_{\psi^{*}}, \quad I=\psi \partial_{\psi}+\psi^{*} \partial_{\psi^{*}}, \quad Z\left(\eta^{0}\right)=\eta^{0} \partial_{\psi}+\eta^{0 *} \partial_{\psi^{*}}
\end{aligned}
$$

the parameters $\tau, \chi$ and $\sigma$ run through the set of real-valued smooth functions of $t$ satisfying the classifying condition (17), and $\eta^{0}$ runs through the solution set of the equation $\mathcal{L}_{V}$.

It is obvious that properties of appropriate subalgebras for the subclass $\mathrm{Sch}_{\mathbb{R}}$ can be obtained by specifying the same properties of appropriate subalgebras for the whole class (11). Thus, inequivalent cases of real-valued potentials admitting Lie symmetry extensions can be singled out from the classification list presented in Table 1. We note, however, that the group classification of real-valued potentials can be easily carried out from the outset.

Theorem 39. A complete list of inequivalent Lie symmetry extensions in the subclass $\mathrm{Sch}_{\mathbb{R}}$ is given in Table 3.

Proof. The proof follows the same pattern as Theorem 24, and we sketch the proof by considering the invariants $k_{1}$ and $k_{2}$. The case $k_{2}=0$ is split into the three subcases $k_{1}=0, k_{1}=1$ and $k_{1} \geqslant 2$. The proof for each subcase is the same as for Theorem 24] except that the parameter $\rho$ in each Lie symmetry vector field satisfies the equation $\rho_{t}=-\frac{1}{4} \tau_{t t}$. If $k_{2}=2$, then the algebra $\mathfrak{g}_{V}^{\text {ess }}$ contains a vector field $Q^{1}=G\left(\chi^{1}\right)+\sigma^{1} M+\rho^{1} I$, where the parameters $\chi^{1}$ and $\sigma^{1}$ are real-valued smooth functions of $t$ with $\chi^{1} \neq 0$ and $\rho^{1}$ is a real constant. Combining $Q^{1}$ with $I$ and using $G^{\sim}$-equivalence, we may assume that $Q^{1}=G(1)$. The equation $\mathcal{L}_{V}$ is invariant with respect to $G(1)$ if and only if the potential $V$ does not depend on $x$. Then the equation $\mathcal{L}_{V}$ is equivalent to the free linear Schrödinger equation. 
Table 3. The classification list for real-valued potentials.

\begin{tabular}{|c|c|c|c|l|}
\hline no. & $k_{1}$ & $k_{2}$ & $V$ & \multicolumn{1}{|c|}{ Basis of $\mathfrak{g}_{V}^{\text {ess }}$} \\
\hline 1 & 0 & 0 & $V(t, x)$ & $M, I$ \\
2 & 1 & 0 & $V(x)$ & $M, I, D(1)$ \\
3 & 3 & 0 & $c x^{-2}, c \in \mathbb{R}_{*}$ & $M, I, D(1), D(t), D\left(t^{2}\right)-\frac{1}{2} t I$ \\
4 & 3 & 2 & 0 & $M, I, D(1), D(t), D\left(t^{2}\right)-\frac{1}{2} t I, G(1), G(t)$ \\
\hline
\end{tabular}

Lie symmetry extensions given in Table 3 are maximal if and only if the potential $V$ does not satisfy an equation of the form (17) in Case 1 and $V \neq b_{2} x^{2}+b_{1} x+b_{0}+c(x+a)^{-2}$ for any real constants $a, b_{0}, b_{1}, b_{2}$ and $c$ in Case 2 .

\section{Conclusion}

In this paper we have completely solved the group classification problem for $(1+1)$-dimensional linear Schrödinger equations with complex-valued potentials. The classification list is presented in Theorem 24 or, equivalently, in Corollary 33. This also gives the group classifications for the larger class of similar equations with variable mass and for the smaller class of such equations with real-valued potentials. We have introduced the notion of uniformly semi-normalized classes of differential equations and developed a special version of the algebraic method of group classification for such classes. This is, in fact, the main result of the paper. The class (1) has the specific property of uniform semi-normalization with respect to linear superposition transformations, which is quite common for classes of homogeneous linear differential equations. Within the framework of the algebraic method, the group classification problem of the class (1) reduces to the classification of appropriate low-dimensional subalgebras of the associated equivalence algebra $\mathfrak{g}^{\sim}$.

We show that the linear span $\mathfrak{g}_{\langle>}$of the vector fields from the maximal Lie invariance algebras of equations from the class (1) is itself a Lie algebra. For each potential $V$, the maximal Lie invariance algebra $\mathfrak{g}_{V}$ of the equation $\mathcal{L}_{V}$ from the class (10) is the semi-direct sum of a subalgebra $\mathfrak{g}_{V}^{\text {ess }}$, of dimension not greater than seven, and an infinite dimensional abelian ideal $\mathfrak{g}_{V}^{\text {lin }}$, which is the trivial part of $\mathfrak{g}_{V}$ and is associated with the linear superposition principle, $\mathfrak{g}_{V}=\mathfrak{g}_{V}^{\text {ess }} \oplus \mathfrak{g}_{V}^{\text {lin }}$. The above representation of $\mathfrak{g}_{V}$ 's yields a similar representation for $\mathfrak{g}_{\langle\rangle}=\sum_{V} \mathfrak{g}_{V}, \mathfrak{g}_{\langle\rangle}=\mathfrak{g}_{\langle\rangle}^{\text {ess }} \in \mathfrak{g}_{\langle\rangle}^{\text {lin }}$, where $\mathfrak{g}_{\langle\rangle}^{\text {ess }}=\sum_{V} \mathfrak{g}_{V}^{\text {ess }}$ is a (finite-dimensional) subalgebra of $\mathfrak{g}_{\langle\rangle}$, and $\mathfrak{g}_{\langle\rangle}^{\text {lin }}=\sum_{V} \mathfrak{g}_{V}^{\text {lin }}$ is its abelian ideal. The projection of the equivalence algebra $\mathfrak{g}^{\sim}$ of the class (1) on the space of variables coincides with $\mathfrak{g}_{\langle\rangle}^{\text {ess }}$. Thus, two objects, $\mathfrak{g}_{\langle\rangle}^{\text {ess }}$ and $\mathfrak{g}^{\sim}$, are directly related to the class (1) and consistent with each other. This is why we classify appropriate subalgebras of $\mathfrak{g}_{\langle\rangle}^{\text {ess }}$ up to $G^{\sim}$-equivalence, each of which coincides with $\mathfrak{g}_{V}^{\text {ess }}$ for some $V$.

The partition into classification cases is provided by two nonnegative integers $k_{1}$ and $k_{2}$, which are characteristic invariants of subalgebras of $\mathfrak{g}_{\langle\rangle}^{\text {ess }}$. This leads to two equivalent classification lists for the potential $V$ depending on which of these invariants is assumed as the leading invariant. The list presented in Table 1 (resp. described in Corollary [33) is constructed under the assumption that the invariant $k_{1}$ (resp. $k_{2}$ ) is leading. Each of the lists consists of eight $G^{\sim}$-inequivalent families of potentials. We have proved that for appropriate subalgebras the invariant $k_{2}$ can take only two values: 0 and 2 , and the invariant $k_{1}$ is not greater than three. Further, the invariant $k_{1}$ cannot equal two for appropriate subalgebras due to the fact that the corresponding subalgebras cannot be maximal Lie symmetry algebras for equations from the class (11). At the same time, the proof of the condition $k_{1} \neq 2$ needs realizing the major part of the group classification of the class under study. 
The cases in the second list for which $k_{2}=0$ coincide with those from the first list. For $k_{2}=2$, the group classification of the class (11) reduces to the group classification of its subclass (12). This subclass is uniformly semi-normalized with respect to a larger family of point symmetry groups than the corresponding groups of linear superposition transformations, which makes the subclass (12) a useful example for group analysis of differential equations. For each equation $\mathcal{L}_{V}$ from the subclass, the essential part $\mathfrak{g}_{V}^{\text {ess }}$ of its maximal Lie invariance algebra $\mathfrak{g}_{V}$ can be written as $\mathfrak{g}_{V}^{\text {ess }}=\mathfrak{g}_{V}^{\text {ext }} \in\left(\mathfrak{g}_{V}^{\text {unf }} \cap \mathfrak{g}_{\langle>}^{\text {ess }}\right)$, where $\mathfrak{g}_{V}^{\text {unf }}$ is an ideal of $\mathfrak{g}_{V}$ and has a similar structure for all equations from the subclass, and $\mathfrak{g}_{V}^{\text {ext }}$ is a subalgebra of $\mathfrak{g}_{V}^{\text {ess }}$. The vector fields from all $\mathfrak{g}_{V}^{\text {ext's of equations }}$ from the subclass (12) constitute the algebra $\mathfrak{g}_{\langle\rangle}^{\text {ext }}$, which is contained in the projection of the equivalence algebra of the subclass (12) and is isomorphic to the algebra $\operatorname{sl}(2, \mathbb{R})$. Therefore, the classification of subalgebras of $\operatorname{sl}(2, \mathbb{R})$ (which is well known) yields the solution of the group classification problem of the subclass (12), whose result is presented in Table 2.

Since the subclass $\mathrm{Sch}_{\mathbb{R}}$ of (1+1)-dimensional linear Schrödinger equations with real-valued potentials is important for applications, we have given its group classification separately by singling out related results from the group classification of the class (11). Since the subclass $\mathrm{Sch}_{\mathbb{R}}$ is also uniformly semi-normalized with respect to linear superposition of solutions, this procedure can be realized within the framework of the algebraic approach by specifying the properties of appropriate subalgebras for the case of real-valued potentials.

Furthermore, the semi-normalization of the above classes of linear Schrödinger equations guarantees that there are no additional point equivalence transformations between classification cases listed for each of these classes.

The new version of the algebraic method that is given in Section 3 and then applied to the symmetry analysis of the class (1) can be regarded as a model for optimizing the group classification of other classes of differential equations (including higher-dimensional cases). We intend to extend our approach to multidimensional linear Schrödinger equations with complexvalued potentials. In this context, it seems that the technique used in the proof of Theorem 24 is more useful for generalizing to the multidimensional case than the alternative proof presented in Section 7 .

\section{Acknowledgements}

The research of C.K. was supported by International Science Programme (ISP) in collaboration with East African Universities Mathematics Programme (EAUMP). The research of R.O.P. was supported by the Austrian Science Fund (FWF), project P25064. The authors are pleased to thank Anatoly Nikitin, Olena Vaneeva and Vyacheslav Boyko for stimulating discussions.

\section{References}

[1] Anderson R.L., Kumei S. and Wulfman C.E., Invariants of the equations of wave mechanics. I, Rev. Mexicana Fís. 21 (1972), 1-33.

[2] Anderson R.L., Kumei S. and Wulfman C.E., Invariants of the equations of wave mechanics. II. One-particle Schroedinger equations, Rev. Mexicana Fís. 21 (1972), 35-57.

[3] Basarab-Horwath P., Güngör F. and Lahno V., Symmetry classification of third-order nonlinear evolution equations. Part I: Semi-simple algebras, Acta Appl. Math. 124 (2013), 123-170.

[4] Basarab-Horwath P., Lahno V. and Zhdanov R., The structure of Lie algebras and the classification problem for partial differential equations, Acta Appl. Math. 69 (2001), 43-94.

[5] Bihlo A., Dos Santos Cardoso-Bihlo E. and Popovych R.O., Enhanced preliminary group classification of a class of generalized diffusion equations, Commun. Nonlinear Sci. Numer. Simul. 16 (2011), 3622-3638, arXiv:1012.0297.

[6] Bihlo A., Dos Santos Cardoso-Bihlo E. and Popovych R.O., Complete group classification of a class of nonlinear wave equations, J. Math. Phys. 53 (2012), 123515, 32 pp., arXiv:1106.4801. 
[7] Bihlo A. and Popovych R.O., Group classification of linear evolution equations, J. Math. Anal. Appl. 448 (2017), 982-1005, arXiv:1605.09251.

[8] Bluman G.W. and Kumei S., Symmetries and differential equations, Springer, New York, 1989.

[9] Bender C.M., Complex Extension of Quantum Mechanics, in Proceedings of Fifth International Conference "Symmetry in Nonlinear Mathematical Physics" (23-29 June, 2003, Kyiv), Proceedings of Institute of Mathematics of NAS of Ukraine 50 (2004), Institute of Mathematics of NAS of Ukraine, Kyiv, pp. 617-628.

[10] Bender C. M., Making sense of non-Hermitian Hamiltonians, Rep. Progr. Phys. 70 (2007), 947-1018.

[11] Boyer C.P., The maximal 'kinematical' invariance group for an arbitrary potential, Helv. Phys. Acta 47 (1974), 589-605.

[12] Doebner H.-D. and Goldin G.A., Properties of nonlinear Schrödinger equations associated with diffeomorphism group representations, J. Phys. A 27 (1994), 1771-1780.

[13] Dos Santos Cardoso-Bihlo E., Bihlo A. and Popovych R.O., Enhanced preliminary group classification of a class of generalized diffusion equations, Commun. Nonlinear Sci. Numer. Simul. 16 (2011), 3622-3638, arXiv:1012.0297.

[14] Fushchich W.I. and Moskaliuk S.S., On some exact solutions of the nonlinear Schrödinger equation in three spatial dimensions, Lett. Nuovo Cimento (2) 31 (1981), 571-576.

[15] Gagnon L. and Winternitz P., Lie symmetries of a generalised non-linear Schrödinger equation. I. The symmetry group and its subgroups, J. Phys. A 21 (1988), 1493-1511.

[16] Gagnon L. and Winternitz P., Lie symmetries of a generalised non-linear Schrödinger equation. II. Exact solutions, J. Phys. A 22 (1989), 469-497.

[17] Gagnon L., Grammaticos B., Ramani A. and Winternitz P., Lie symmetries of a generalised non-linear Schrödinger equation. III. Reductions to third-order ordinary differential equations, J. Phys. A 22 (1989), 499-509.

[18] Gagnon L. and Winternitz P., Exact solutions of the cubic and quintic nonlinear Schrödinger equation for a cylindrical geometry, Phys. Rev. A (3) 39 (1989), 296-306.

[19] Gagnon L. and Wintenitz P., Symmetry classes of variable coefficient nonlinear Schrödinger equations, J. Phys. A 26 (1993), 7061-7076.

[20] Gazeau J.P. and Winternitz P., Symmetries of variable coefficient Korteweg-de Vries equations, J. Math. Phys. 33 (1992), 4087-4102.

[21] Lie S., Über die Integration durch bestimmte Integrale von einer Klasse linear partieller Differentialgleichung, Arch. for Math. 6 (1881), 328-368. (Translation by N.H. Ibragimov: Lie S., On integration of a class of linear partial differential equations by means of definite integrals, CRC Handbook of Lie Group Analysis of Differential Equations, vol. 2, CRC Press, Boca Raton, 1994, pp. 473-508.)

[22] Lisle I.G., Equivalence transformations for classes of differential equations, PhD. thesis, University of British Columbia, 1992.

[23] Magadeev B.A., Group classification of nonlinear evolution equations, Algebra i Analiz 5 (1993), 141-156 (in Russian); English translation in St. Petersburg Math. J. 5 (1994), 345-359.

[24] Miller W., Symmetry and separation of variables, Addison-Wesley Publishing Co., Reading, MA, 1977.

[25] Mostafazadeh A., A dynamical formulation of one-dimensional scattering theory and its applications in optics, Ann. Physics 341 (2014), 77-85.

[26] Nattermann P. and Doebner H.-D., Gauge classification, Lie symmetries and integrability of a family of nonlinear Schrödinger equations, J. Nonlinear Math. Phys. 3 (1996), 302-310.

[27] Niederer U., The maximal kinematical invariance group of the free Schrödinger equation, Helv. Phys. Acta. 45 (1972), 802-810.

[28] Niederer U., The maximal kinematical invariance group of the harmonic oscillator, Helv. Phys. Acta. 46 (1973), 191-200.

[29] Niederer U., The group theoretical equivalence of the free particle, the harmonic oscillator and the free fall, in Proceedings of the 2nd International Colloquium on Group Theoretical Methods in Physics, University of Nijmegen, The Netherlands, 1973.

[30] Niederer U., The maximal kinematical invariance groups of Schrödinger equations with arbitrary potentials, Helv. Phys. Acta. 47 (1974), 167-172.

[31] Olver P.J., Applications of Lie groups to differential equations, Springer-Verlag, New York, 1993. 
[32] Opanasenko S., Bihlo A. and Popovych R.O., Group analysis of general Burgers-Korteweg-de Vries equations, J. Math. Phys. 58 (2017), 081511, 37 pp., arXiv:1703.06932

[33] Ovsiannikov L.V., Group properties of nonlinear heat equation Dokl. AN SSSR 125 (1959), 492-495 (in Russian).

[34] Ovsiannikov L.V., Group analysis of differential equations, Academic Press, New York, 1982.

[35] Ovsjannikov L.V. and Ibragimov N. H., Group analysis of the differential equations of mechanics, in General mechanics, vol. 2, Akad. Nauk SSSR Vsesojuz. Inst. Nauchn. i Tehn. Informacii, Moscow, 1975, pp. 5-52 (in Russian).

[36] Popovych R.O. and Ivanova N.M., New results on group classification of nonlinear diffusion-convection equations, J. Phys. A 37 (2004), 7547-7565, arXiv:math-ph/0306035.

[37] Popovych R.O. and Ivanova N.M. and Eshraghi H., Lie symmetries of (1+1)-dimensional cubic Schrödinger equation with potential, Proceedings of Institute of Mathematics of NAS of Ukraine 50 (2004), 219-224, arXiv:math-ph/0310039.

[38] Popovych R.O., Ivanova N.M and Eshragi H., Group classfication of (1+1)-dimensional Schrödinger equations with potentials and power nonlinearities, J. Math. Phys 45 (2004), 3049-3057, arXiv:math-ph/0311039

[39] Popovych R.O., Kunzinger M. and Eshragi H., Admissible transformations and normalized classes of nonlinear Schrödinger equations, Acta Appl. Math. 109 (2010), 315-359, arXiv:math-ph/0611061

[40] Popovych R.O., Kunzinger M. and Ivanova N.M., Conservation laws and potential symmetries of linear parabolic equations, Acta Appl. Math. 100 (2008), 113-185, arXiv:0706.0443.

[41] Vaneeva O.O., Popovych R.O. and Sophocleous C., Enhanced group analysis and exact solutions of variable coefficient semilinear diffusion equations with a power source, Acta Appl. Math. 106 (2009), 1-46, arXiv:0708.3457.

[42] Vaneeva O.O., Popovych R.O. and Sophocleous C., Extended group analysis of variable coefficient reaction-diffusion equations with exponential nonlinearities, J. Math. Anal. Appl. 396 (2012), 225-242, arXiv:1111.5198.

[43] Zhdanov R. and Roman O., On preliminary symmetry classification of nonlinear Schrödinger equations with some applications to Doebner-Goldin models, Rep. Math. Phys. 45 (2000), 273-291.

[44] Zhdanov R.Z. and Lahno V.I., Group classification of heat conductivity equations with a nonlinear source, J. Phys. A 32 (1999), 7405-7418. 